\title{
Standard model baryogenesis through four-fermion operators in braneworlds
}

\author{
Daniel J. H. Chung* \\ CERN Theory Division, CH-1211 Geneva 23, Switzerland \\ Thomas Dent ${ }^{\dagger}$ \\ Michigan Center for Theoretical Physics, University of Michigan, Ann Arbor, Michigan 48109
}

(Received 7 February 2002; published 26 June 2002)

\begin{abstract}
We study a new baryogenesis scenario in a class of braneworld models with low fundamental scale, which typically have difficulty with baryogenesis. The scenario is characterized by its minimal nature: the field content is that of the standard model and all interactions consistent with the gauge symmetry are admitted. Baryon number is violated via a dimension-6 proton decay operator, suppressed today by the mechanism of quark-lepton separation in extra dimensions; we assume that this operator was unsuppressed in the early Universe due to a time-dependent quark-lepton separation. The source of $C P$ violation is the CKM matrix, in combination with the dimension-6 operators. We find that almost independently of cosmology, sufficient baryogenesis is nearly impossible in such a scenario if the fundamental scale is above $100 \mathrm{TeV}$, as required by an unsuppressed neutron-antineutron oscillation operator. The only exception producing sufficient baryon asymmetry is a scenario involving out-of-equilibrium $c$ quarks interacting with equilibrium $b$ quarks.
\end{abstract}

DOI: 10.1103/PhysRevD.66.023501

PACS number(s): $98.80 . \mathrm{Cq}, 11.10 . \mathrm{Kk}, 95.30 . \mathrm{Cq}$

\section{INTRODUCTION}

The experimental lower limit on the lifetime of the proton is a severe problem for models in which the fundamental scale of quantum gravity is low compared to the supersymmetric grand unified theory (GUT) scale $10^{16} \mathrm{GeV}[1,2]$. A baryon number U(1) symmetry cannot be gauged in field theory; like other accidental symmetries it is expected to be violated by effects at the string scale or by quantum wormholes and virtual black holes [3]. Such $B$ violation would appear at low energies as nonrenormalizable operators, for example of the form $\lambda q q q l / M_{f}^{2}$ where $M_{f}$ is the fundamental scale and $\lambda$ is expected to be $\mathcal{O}(1)$ in the absence of suppression mechanisms. Then for $M_{f}$ in the $\mathcal{O}(10-100) \mathrm{TeV}$ range, for which collider signals of the fundamental degrees of freedom or of large extra dimensions may be observable, $\tau_{p}$ comes out to be under a second, to be compared with the (mode-dependent) experimental bounds of order $10^{32}$ years [4]. Various solutions have been proposed [5-7] all of which have implications for the production of an excess of baryons over antibaryons in the early Universe, for which $B$ violation is a precondition.

The production of gravitational Kaluza-Klein (KK) modes in the early Universe in such models, in which some compactified extra dimensions are orders of magnitude larger than the fundamental length, gives a severe upper bound on the maximum temperature attained consistent with cosmological observations. Even for the maximum number (usually considered as 6) of large extra dimensions and the relatively large value $M_{f}=100 \mathrm{TeV}$, a maximum temperature of a few $\mathrm{GeV}$ is the upper bound if overclosure of the Universe, disruption of the successful predictions of nucleosynthesis, and an observationally unacceptable level of background gamma

\footnotetext{
*Email address: daniel.chung@ cern.ch

†Email address: tdent@umich.edu
}

rays from KK mode decay are to be avoided [5,8]. The energy density in KK modes would also affect the evolution of density fluctuations by altering the time of matter-radiation equality [9]. Astrophysical production and decay of such modes also leads to an independent lower bound on the fundamental scale [10], which is also constrained by the nonobservation (to date) of direct and loop effects in experiments [11].

Any attempt at explaining proton longevity and baryogenesis should operate within these constraints. Exact (anomalyfree) discrete or horizontal symmetries can be imposed to forbid $B$-violating operators mediating proton decay $[5,12]$ while allowing others, through which baryogenesis occurs: this approach requires an " $X$ boson" to be present, with couplings which appear unnaturally small (in contrast with the standard GUT or leptogenesis scenarios). Baryon number can be gauged if the anomaly is canceled by a string theory mechanism, or $B$ violation may be forbidden to all orders in perturbation theory by string selection rules, in some "intersecting brane" models [7]. Note however that in a more general class of intersecting brane models [13], such selection rules do not prevent the four-fermion operators from appearing, as discussed above, in which case the fundamental scale cannot be low.

If baryon number is perturbatively exact, nonperturbative processes [14] are the only option to create net baryon number. It is difficult to see how this proposal can be reconciled with cosmological constraints, since any such processes would operate at or above the electroweak scale and be enormously suppressed at low temperature [15].

A novel mechanism for suppressing proton decay in the context of a low fundamental scale theory has been proposed in Ref. [6]. This is a geometrical mechanism for suppressing 4D $B$-violating operators, namely localization of fermions in extra dimensions. The simplest implementation is for the $\mathrm{SU}(2)_{L} \times \mathrm{U}(1)_{Y}$ gauge fields to propagate in one extra dimension (cf. [16]), in which the quark and lepton wave func- 


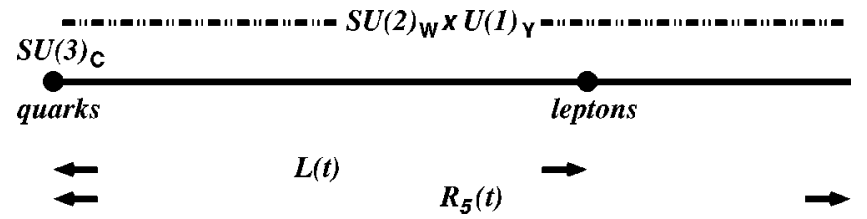

FIG. 1. Schematic setup of our braneworld.

tions are peaked about points separated by a distance $L$ $\sim 30 M_{f}^{-1}$ (Fig. 1). Then any strong $B$-violating operators in the effective $5 \mathrm{D}$ theory can only produce proton decay proportional to the overlap of the wave functions, which can be exponentially small. Alternatively, proton decay by exchange of massive modes is suppressed by the Yukawa propagator over the distance $L[17]$. Nonperturbative quantum effects which may lead to proton decay, for example virtual black holes [18], are also exponentially suppressed due to the integration over the fifth dimension.

Baryogenesis in such scenarios has been discussed in Ref. [19]. However, the authors of that work introduced a standard model singlet scalar with renormalizable baryon number-violating interactions and primarily considered the effect of thermal corrections to the wave function overlap which controls the strength of the baryon number violation.

An Affleck-Dine-type scenario consistent with large extra dimensions, which involves introducing a scalar charged under a new $U(1)$ group, has recently been described in [20]; while the idea appears viable from a cosmological viewpoint, the authors do not discuss how $B$-violating couplings which induce proton decay are to be suppressed, while still keeping the operators by which the scalar decays. One might imagine that additional exact symmetries could be imposed to achieve this, in the spirit of [5].

In this work, we consider a related, but simpler class of scenarios and report mostly on a "no go" result. Specifically, we consider the feasibility of baryogenesis under the following conditions:

(1) The only renormalizable operators that are present at the electroweak scale are those of the standard model, and the perturbative 4D effective field theory (dimensionally reduced from an intrinsically higher-dimensional theory) is valid. (The possibility of doing without an " $X$ boson" was briefly mentioned in [19].) In other words, we work with an effective theory with the top quark as the heaviest field that has not been integrated out and neglect any Lorentz violating operators resulting from time dependence of the extra dimensional geometry. This condition is motivated from our ignorance about the physics beyond the standard model and the fact that no viable baryogenesis scenario has yet been proposed with only the standard model fields and gravity.

(2) The leading baryon number-violating operator due to physics at the fundamental scale is a dimension- 6 operator of the form

$$
\frac{v_{s}}{M_{f}^{2}} q q q l
$$

where $v_{s}$ is the wave function overlap, which is a time dependent function. The wave function overlap $v_{s}$ can be time- dependent whenever fermion localization is controlled by a field, akin to the radion, which is initially displaced from the minimum of its effective potential. The work of [19] can also be taken to be of this type, where the "radion" is always at the minimum of a time dependent effective potential, which is derived from time dependent finite temperature effects. Note that this condition implies the assumption that 4D instanton or sphaleron induced baryon number violating operators are weaker than the dimension- 6 operator, i.e., only perturbative physics is analyzed for dimension 4 operators. This is a reasonable assumption as long as the temperature is well below the electroweak scale (which is required anyway in low fundamental scale models) and the $\mathrm{SU}(2)_{L}$ coupling remains small (Appendix B).

(3) The 4D Planck scale $M_{p l}$ has an adiabatic time dependence due to the change in the volume of extra dimensions, and the fundamental scale of gravitational physics is at $M_{f}$ $\simeq 10^{5} \mathrm{GeV}$. The upper bound on this scale comes from the desire to ameliorate the hierarchy problem without supersymmetry (SUSY), while the lower bound is obtained by the bounds on neutron-antineutron oscillations, which cannot be suppressed by a geometrical mechanism. Note that there is still a certain amount of fine tuning, of order $10^{3}$, with such a (relatively) large fundamental scale.

(4) There is no electroweak phase transition because of a low reheating temperature (i.e., $T<30 \mathrm{GeV}$ ) [5,8]. Baryogenesis is also assumed to occur above the QCD phase transition temperature (i.e., $T>0.2 \mathrm{GeV}$ ).

(5) The effective 3D space is homogeneous and isotropic at all times when the temperature of the universe is $T$ $>0.2 \mathrm{GeV}$.

In this context, we analyze the possibility of baryogenesis and find specific conditions under which it is feasible.

Our setup contains all the necessary ingredients of baryogenesis [21]:

(1) Baryon number violation (dimension-6 operator).

(2) $C$ and $C P$ violation [standard model CabibboKobayashi-Maskawa (CKM) + dimension-6 operator]-the resulting quantity is much larger than the Jarlskog invariant.

(3) Nonequilibrium (spacetime expansion or other unspecified means).

The reason for the difficulty of this scenario will be ingredient (1), the baryon number violating operator being too weak in the low temperature setting, independently of ingredients (2) and (3). Since the usual Jarlskog invariant of the $\mathrm{SM}$ is not the relevant quantity, given the presence of dimension- 6 operators, the reason why this baryogenesis scenario is difficult has nothing to do with traditional wisdom. In particular, we find that dimension- 6 operators are in most cases too weak to compete with baryon number-conserving operators which create entropy, for most sets of reactants.

Naively, one finds this result a little surprising because one would expect

$$
\eta_{B} \equiv \frac{n_{B}}{s} \sim \frac{\delta_{C P V} B n}{g_{* S} T_{*}^{3}}
$$

can be easily engineered to be sufficiently large, where $\delta_{C P V}$ is the dimensionless quantity associated with $C P$ violation, $B$ 
is the maximal branching ratio into the baryon number violating channel fixed by our model, $n$ is the number density of particles that can be converted into baryon number, $g_{* S}$ is the number of degrees of freedom contributing to the entropy, and $T_{*}$ is the temperature at some fiducial time after which the baryon number is conserved. However, even though $\delta_{C P V}$ can be naturally large in our scenario (not governed by the Jarlskog invariant), $B n /\left(g_{* S} T_{*}^{3}\right)$ cannot in general be made large because as the baryon number violating reactions are creating baryon asymmetry, the baryon number conserving counterparts are creating proportionately large amounts of entropy density. In other words, having the quark-lepton separation go to zero does not generate order 1 branching of the baryon number violating channels. Instead, such a channel is always suppressed by a scale much larger than the electroweak scale.

We do find an exception to our "no go" rule for a special situation in which the universe starts out dominated by the $b$ and $c$ quarks and gluons (which might happen through the peculiarities of reheating after inflation), and the lighter quark $c$ is out of equilibrium while the heavier quark $b$ is in equilibrium, for example due to the anomalously large expansion rate and the anomalously small density of $c$ quarks. In this exceptional scenario, there are two key ingredients compensating the suppression of the dimension 6 operator: (1) Due to the small $\left|V_{c b}\right|^{2}$, the $b$ quark interactions with outof-equilibrium $c$ quarks do not generate significant entropy; (2) the integration time for baryogenesis can be made long if the expansion rate is small, due to the nonstandard cosmology of extra dimensions. However, this remote possibility, which requires additional assumptions about special initial conditions and/or more new physics, is unlikely to be realizable, and we do not have a detailed model to demonstrate its full viability.

Throughout this paper, we do not assume thermal equilibrium initial conditions, except where explicitly noted. Hence, although the short distance operators are fixed, the nonzero initial particle densities that exist in the universe can be $a$ priori arbitrary combinations of species. Thus, when we consider bounds, we explicitly check all likely combinations of species without any bias to their initial number distributions, except when otherwise noted. As far as the out-ofequilibrium condition due to the expansion of the universe is concerned, we assume that somehow the Planck scale can be adjusted to dial into the appropriate expansion rate. Of course, in a more completely specified model, this may turn out to be problematic, due to the bounds on moduli overproduction and other light moduli problems. However, our results are robust with respect to how the moduli problems and initial conditions problems can be solved. In fact most of our results do not depend on how the particles are out of equilibrium. This broad insensitivity with respect to cosmological details is one of the key features to our work.

The order of our presentation will be as follows. We begin with a discussion of the class of braneworlds and the energy scales and couplings that we are interested in. We then present our constraint on this scenario for baryogenesis with dimension- 6 operators in the low temperature regime. Finally, we summarize and conclude.

\section{BRANEWORLD SETUP AND SCALES}

The simplest way to realize the proposal of geometrical proton decay suppression is for the "brane" on which we live to be a nontrivial scalar field profile in an extra dimension [22]. On a smooth manifold, chiral fermions are localized in the presence of a scalar field profile with Yukawa couplings to the fermion fields, resulting in a positiondependent effective mass term. The simplest ansatz is to take a linearly varying scalar field, which results in chiral fermion zero modes with a Gaussian wave function, localized about the point where the effective fermion mass vanishes [6]. On an orbifold [23] one may impose special boundary conditions, which themselves ensure that the lowest mode is chiral, then with a scalar field which is forced to take up a kink configuration one obtains a fermion wave function which varies approximately as $\cosh ^{-2}\left(k x_{5}\right)$.

By coupling the leptons and quarks differently to different scalar fields, one may separate the zero mode wave functions such that the wave function overlap factor $v_{s}$ in Eq. (1) obtains an exponentially suppressed value. As discussed in [23], it is not easy to induce large displacements of the points of localization away from special points such as the orbifold fixed points (whose boundary conditions force the zero of the scalar field) without introducing arbitrary scale parameters. In this paper, we will not assume any particular mechanism to make these localization points (which we will sometimes loosely call "branes") dynamical, but rather suppose that large dynamical displacements of the localization points (branes) from their final value are possible. Hence, we treat $v_{s}$ as merely a time-dependent function that is controlled by an exponential of the form

$$
v_{s} \sim e^{-\zeta M_{f} L}
$$

where $\zeta$ is a constant coefficient of order 1 and $L$ is a timedependent function giving the distance between the quark and lepton localizations. There may be additional contributions to $v_{s}$ varying as $e^{-(\mu L)^{2}}$ but these will be subleading unless $\mu^{2} \ll M_{f}^{2}$. We will see below that $\zeta$ actually has to be greater than or equal to about $3 / 2$ in our scenario.

The baryogenesis scenario is then as follows: at some early time in the evolution of the Universe, $M_{f} L \sim \mathcal{O}(1)$ and the baryon number violating operator is suppressed only by the fundamental scale. By the time of nucleosynthesis, $\zeta M_{f} L$ will have settled down to today's value of about 50, consistent with proton decay constraints (see Appendix A). For the purposes of our paper, we will not need the specific details of how the quark-lepton separation is stabilized at its final value. We will show that the short-distance physics prevents sufficient baryogenesis in the minimal model that we consider, except in the special case already mentioned.

Since the zero mode quark and lepton wave functions are separated in the extra dimension and since the quarks and the leptons interact through the $S U(2)_{L} \times U(1)_{Y}$ gauge group, these gauge fields must also propagate in the extra dimension, and the 4D gauge theory must come from dimensional reduction. Similarly, since everything interacts with gravity, 4D gravity also arises from dimensional reduction. We now 
derive bounds on the fundamental scale as well as the size of the extra dimensions by demanding perturbativity of gauge fields propagating in the extra dimensions and that Einstein gravity be recovered [24].

From gravitational dimensional reduction, given that there are a total of $n$ extra dimensions and one of them is asymmetric with length $R_{5}\left(t_{3}\right)$, the others having lengths $R_{i}, i$ $=1, \ldots, n-1$, we have

$$
M_{f}^{2}=\frac{\bar{M}_{p l}^{2}}{\left(R_{5}\left(t_{3}\right) M_{f}\right) \prod_{i}^{n-1}\left(R_{i} M_{f}\right)}
$$

where $t_{3}$ denotes the present time and $\bar{M}_{p l}$ is the reduced Planck mass $M_{p l} / \sqrt{8 \pi}$. There is also a perturbativity constraint on the Yang-Mills coupling constant. In $4+p$ dimensions [for example for a $(3+p)$-brane], the Yang-Mills coupling is

$$
g_{4+p}^{2}=\frac{\lambda_{4+p}^{2}}{M_{f}^{p}}
$$

where $\lambda_{4+p}$ is a dimensionless number. By dimensional reduction to $4 \mathrm{D}$, we have the 4-dimensional dimensionless Yang-Mills coupling $g_{4}$ related to $\lambda_{4+p}$ by

$$
\lambda_{4+p}^{2}=g_{4}^{2}\left(R_{5} M_{f}\right) \prod_{i}^{p-1}\left(R_{i} M_{f}\right) .
$$

If $g_{4}(t)$ is to remain perturbative at time $t_{1} \leqslant t_{3}$, we must have

$$
\left(\frac{g_{4}^{2}\left(t_{3}\right)}{4 \pi}\right)\left(\prod_{i}^{p-1}\left(\frac{R_{i}\left(t_{3}\right)}{R_{i}\left(t_{1}\right)}\right)\right)\left(\frac{R_{5}\left(t_{3}\right)}{R_{5}\left(t_{1}\right)}\right)<1 .
$$

Given that $g_{4}^{2}\left(t_{3}\right) /(4 \pi) \approx 1 / 31$ for the EW theory $(\alpha$ $\left.\equiv e^{2} / 4 \pi \simeq 1 / 137=\alpha_{W} \sin ^{2} \theta_{W} \simeq 0.225 \alpha_{W}\right)$, we require

$$
R_{5}\left(t_{3}\right) / R_{5}\left(t_{1}\right)\left(\prod_{i}^{p-1}\left(\frac{R_{i}\left(t_{3}\right)}{R_{i}\left(t_{1}\right)}\right)\right)<31 \text {. }
$$

If we have a scenario in which

$$
R_{5}(t)=\text { const }
$$

this is not an issue, but in general, this is constraining.

Now, let us consider absolute perturbativity of the five dimensional theory. Since the cutoff is set at $M_{f}$, perturbativity requires

$$
\frac{g_{4+p}^{2}}{4 \pi} M_{f}^{p}<1
$$

Using Eq. (5), we obtain

$$
\frac{\lambda_{4+p}^{2}}{4 \pi}=\frac{g_{4}^{2}\left(t_{3}\right)}{4 \pi}\left(R_{5}\left(t_{3}\right) M_{f}\right) \prod_{i}^{p-1}\left(R_{i}\left(t_{3}\right) M_{f}\right)<1
$$

for $5 \mathrm{D}$ perturbativity. However, this requires the volume enhancement factor to be small. In the end, if we want to minimize this effect, we must have only a D4-brane (i.e., $p=1$ ). Note that our perturbativity constraint is for calculational feasibility rather than necessarily for fundamental physical reasons.

Coming back to Eq. (4), if we impose that $R_{5}\left(t_{3}\right)$ in which the gauge fields propagate is the largest extra dimension

$$
R_{i}<R_{5}\left(t_{3}\right) \approx \frac{1}{\alpha_{W} M_{f}}
$$

where the approximate equality is due to Eq. (6), one sees that

$$
M_{f}>\bar{M}_{p l} \alpha_{W}^{n / 2} \approx 1.5 \times 10^{13} \mathrm{GeV}
$$

To restate this, if the gauge fields come from dimensional reduction of the largest compact dimension, perturbativity requires

$$
M_{f}>10^{13} \mathrm{GeV}
$$

Hence, for this scenario to have $M_{f} \leqslant 10^{5} \mathrm{GeV}$, there must be compact dimensions larger than the dimension in which the gauge fields propagate.

Then we take, for simplicity, 5 large extra dimensions of length $R^{\prime}$ in which the gauge fields do not propagate, and one comparatively smaller extra dimension of length $R_{5}\left(t_{3}\right)=1 / \alpha_{W} M_{f}$ in which (electroweak) gauge fields do propagate. Since in that case,

$$
M_{f}^{2}=\frac{\alpha_{W} \bar{M}_{p l}^{2}}{\left(R^{\prime} M_{f}\right)^{5}}
$$

we can choose

$$
\begin{aligned}
R^{\prime} & =\frac{2 \times 10^{5}}{M_{f}}\left(\frac{10^{5} \mathrm{GeV}}{M_{f}}\right)^{2 / 5} \\
& =2 \mathrm{GeV}^{-1}\left(\frac{10^{5} \mathrm{GeV}}{M_{f}}\right)^{2 / 5}
\end{aligned}
$$

Furthermore, the experimental limit from neutronantineutron oscillations forces $M_{f} \geqslant 10^{5} \mathrm{GeV}$ [5]; therefore $R^{\prime}$ must be smaller than about $2 \mathrm{GeV}^{-1}$. In the spirit of the original motivation for large extra dimensions, namely in order to ameliorate the hierarchy problem, we take the smallest consistent value of $M_{f}$ and set it to $M_{f} \approx 10^{5} \mathrm{GeV}$.

What is important to keep in mind from this section is Eq. (6). Since $\alpha_{W}\left(t_{3}\right) \equiv g_{4}^{2}\left(t_{3}\right) / 4 \pi \approx 1 / 31$, the maximum value that $V\left(t_{3}\right) M_{f}$ can take is 31 . Hence, proton decay constraint requires $\zeta \geqslant 3 / 2$ as claimed below Eq. (3). Furthermore, to "unsuppress" the dimension-6 operator, $L M_{f} \approx 1$ is required during baryogenesis. This means that if the distance between the branes $L$ is equal to the time dependent size of the extra dimension $R_{5}$, then $\alpha_{W} \approx 1$ during baryogenesis. However, as shown in Fig. 1, if the distance between the branes can be adjusted independently of the size of the extra dimension, 
this need not be true. Finally, if we require that no KK modes of the $S U(2)_{L} \times U(1)_{Y}$ gauge group contribute to the baryogenesis dynamics, we must have $R_{5}^{-1}>100$. This leads to the requirement that $\alpha_{W}(t)>10^{-3}$. We will henceforth take $\alpha_{W}$ to be a free (time-dependent) parameter, with a value

$$
10^{-3}<\alpha_{W}(t)<1 .
$$

With a setup in which proton decay and other $B$-violating processes are suppressed by the quark-lepton separation, the next question to ask is which processes dominated at the epoch when $L$ was smaller $\left(L^{-1} \sim M_{f}\right)$. Proton decay due to massive fermion exchange, which appears in the effective theory by operators suppressed as $e^{-k L M_{f}}$ with $k \sim 1$, is the most "difficult" to suppress at the present epoch, since one requires $M_{f} L \geqslant 50$ at the present time up to factors of order 1 , and $R$ is bounded from above by $\alpha_{W}^{-1}$ if $\mathrm{SU}(2)_{\mathrm{W}}$ is to remain perturbative in the bulk. Decay mediated by local interactions in the $d=5$ effective field theory, requiring the localized SM fermions to propagate through the bulk with a suppression of $e^{-\mu^{2} L^{2}}$, might also be problematic if $\mu^{2}$ is small relative to $M_{f}^{2}$, as it must be for a credible field theory model of localization. Here we would require $\mu^{2} L^{2} \geqslant 50$ today. Nonperturbative effects involving the extra dimensions should also be considered, for example virtual black holes or wormholes connecting the two branes. However, given very reasonable assumptions for the topology of the corresponding instantons, their action is large enough for the effects to be negligible [6]. All other $R$-dependent effects turn out generically to be smaller, including the weak instantons which do not cause proton decay but might be important in baryogenesis (Appendix B). Then, assuming that both suppression factors saturate the current bound, as $R$ shrinks from its present value the effective operators suppressed by $e^{-\mu^{2} L^{2}}$ grow much more quickly than those varying as $e^{- \text {const } M_{f} R}$. At the time of baryogenesis, for $R$ comparable to $M_{f}$, the dominant processes (all other things being equal) will be those mediated by the SM fermions propagating through the bulk, for which the suppression is $\sim e^{-\mu^{2} M_{f}^{2}} \sim 1$.

The goal now is to see if, without an electroweak phase transition, we can create enough baryon asymmetry with the dimension-6 operator which is unsuppressed.

\section{BARYOGENESIS}

The defining characteristic of our class of baryogenesis scenarios is the leading baryon number-violating dimension- 6 operator, one example of which is written in the gauge eigenstate basis as

$$
\begin{aligned}
\Delta L= & \frac{v_{s} \lambda^{a n b r}}{M_{f}^{2}}\left(Q_{L}^{a}\right)_{i}^{T} C^{-1}\left(Q_{L}^{n}\right)_{j}\left(L_{L}^{b}\right)_{l}^{T} C^{-1}\left(Q_{L}^{r}\right)_{m} \\
& \times\left[c_{1} \epsilon^{i j} \epsilon^{l m}+c_{2} \epsilon^{i l} \epsilon^{j m}+c_{3} \epsilon^{i m} \epsilon^{j l}\right]
\end{aligned}
$$

where we have suppressed color indices, $\lambda^{a n b r}$ are familydependent coefficients, $\{i, j, l, m\}$ are $S U(2)_{L}$ indices, summation of indices is implicit, and $c_{1}, c_{2}$ and $c_{3}$ are constant real coefficients. The wave function overlap integral that

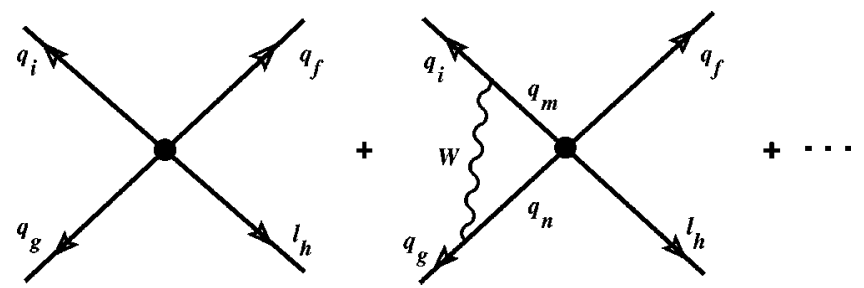

FIG. 2. Diagrams interfering for baryogenesis.

gives rise to $v_{s}$ will be taken to be 1 during baryogenesis. Rotating the quarks and leptons into mass eigenstates, we have

$$
\begin{aligned}
& \left(Q^{a}\right)_{i}=U_{i}^{a \tilde{a}}\left(\widetilde{Q}^{\tilde{a}}\right)_{i} \\
& \left(L^{a}\right)_{i}=W_{i}^{a \tilde{a}}\left(\widetilde{L^{a}}\right)_{i}
\end{aligned}
$$

which corresponds to the usual flavor mixing matrices $V_{\mathrm{CKM}}=U_{u}^{\dagger} U_{d}$ and $V_{M N S}=W_{u}^{\dagger} W_{d}$. This then gives

$$
\begin{aligned}
\Delta L= & \frac{\lambda_{i j m l}^{a n b r}}{M_{f}^{2}}\left(\widetilde{Q}_{L}^{a}\right)_{i}^{T} C^{-1}\left(\widetilde{Q}_{L}^{n}\right)_{j}\left(\widetilde{L}_{L}^{b}\right)_{l}^{T} C^{-1}\left(\widetilde{Q}_{L}^{r}\right)_{m} \\
& \times\left[c_{1} \epsilon^{i j} \epsilon^{l m}+\cdots\right]
\end{aligned}
$$

where

$$
\lambda_{i j l m}^{a n b r}=\lambda^{a^{\prime} n^{\prime} b^{\prime} r^{\prime}} U_{i}^{a^{\prime} a} U_{j}^{n^{\prime} n} U_{m}^{r^{\prime} r} W_{l}^{b^{\prime} b} .
$$

Note that due to the introduction of the dimension- 6 operators, the global symmetry of the theory (in the limit that the Yukawa couplings vanish) is different from the usual low energy analysis of the standard model. Hence, we have more than one rephasing invariant $C P$-violating complex parameter.

Now consider for example the interference between the tree level and the one-loop diagrams shown in Fig. 2, assuming for simplicity that $c_{3}=c_{1}=0$ and $c_{2}=1$. If the tree level amplitude is denoted as $\mathcal{M}_{1}$ while the one loop amplitude is labeled as $\mathcal{M}_{2}$, we know that $\mathcal{M}_{1}$ can be written as

$$
\mathcal{M}_{1}=R_{1} \lambda_{1122}^{a t b s}
$$

where $R_{1}$ is purely real, while $\mathcal{M}_{2}$ will in general be of the form

$$
\mathcal{M}_{2}=\left(R_{2}+i I_{2}\right) V_{i s} V_{t j}^{*} \lambda_{1122}^{a j b i}
$$

where $R_{2}$ and $I_{2}$ are real functions. Note that $I_{2}$ will in general arise if we can cut the propagators in the loop (to make two tree level graphs) and put them on shell simultaneously. For the $C P$ conjugate reaction $\mathcal{M}_{\overline{1}}=R_{1} \lambda_{1122}^{a t b s *}=\mathcal{M}_{1}^{*}$, but

$$
\mathcal{M}_{\overline{2}}=\left(R_{2}+i I_{\overline{2}}\right) V_{i s}^{*} V_{t j} \lambda_{1122}^{a j b i *} \neq \mathcal{M}_{2}^{*}
$$

where in particular, the imaginary part arising from the cut propagators is not the complex conjugate of that for the $C P$-conjugate reaction, since it derives from kinematics alone. 
In the Boltzmann equations for the baryon number density, the probability of interest will be

$$
\begin{aligned}
D & \equiv\left\langle\left|\mathcal{M}_{q l \rightarrow \bar{q} q}\right|^{2}\right\rangle-\left\langle\left|\mathcal{M}_{\bar{q} \bar{l} \rightarrow q q}\right|^{2}\right\rangle \\
& =2 \operatorname{Re}\left[\left\langle\mathcal{M}_{1} \mathcal{M}_{2}^{*}-\mathcal{M}_{\overline{1}} \mathcal{M}_{\frac{2}{2}}^{*}\right\rangle\right] \\
& =4\left\langle R_{1}\left(I_{2}+I_{2}\right)\right\rangle \delta
\end{aligned}
$$

where we have defined

$$
\delta \equiv \operatorname{Im}\left[V_{i s}^{*} V_{t j} \lambda_{1122}^{a j b i *} \lambda_{1122}^{a t b s}\right]
$$

and the \langle\rangle represents spin averaging. Written out explicitly, this interference term is

$$
\begin{aligned}
D= & \frac{-g^{2}}{4 \pi M_{f}^{4}} \sum_{i j} \operatorname{Im}\left\{\lambda_{1122}^{a j b i *} \lambda_{1122}^{a t b s} V_{i s} V_{t j}^{*}\right\} I_{s t i j}^{\alpha \beta} \\
& \times \operatorname{Re}\left\{\operatorname{Tr}\left[\not p_{3} \gamma^{\nu} \gamma_{\alpha} \not_{1} \not_{4} \gamma_{\nu} \gamma_{\beta} \not_{2} P_{R}\right]\right\} \\
& + \text { parity odd }
\end{aligned}
$$

where the external momenta $p_{i}$ are labeled clockwise and constrained to satisfy $p_{1}+p_{2}-p_{3}-p_{4}=0, P_{R}=\left(1+\gamma_{5}\right) / 2$, and

$$
\begin{aligned}
I_{s t i j}^{\alpha \beta} \equiv & \operatorname{Re}\left\{2 \pi \int \frac{d^{4} k}{(2 \pi)^{4}}\left[\frac{\left(p_{3}-k\right)^{\alpha}}{\left(p_{3}-k\right)^{2}-m_{u_{i}}^{2}}\right]\right. \\
& \left.\times\left[\frac{\left(p_{4}+k\right)^{\beta}}{\left(p_{4}+k\right)^{2}-m_{d_{j}}^{2}}\right] \frac{1}{k^{2}-M_{W}^{2}}\right\} .
\end{aligned}
$$

Note that $I_{s t i j}^{\alpha \beta}$ vanishes only if the intermediate quark states cannot go on shell. If the intermediate quark states can go on shell, the expression becomes

$$
\begin{aligned}
I_{s t i j}^{\alpha \beta}= & -8 \pi^{3} \int \frac{d^{3} q_{1}}{(2 \pi)^{3} 2 E_{q_{1}}} \frac{d^{3} q_{2}}{(2 \pi)^{3} 2 E_{q_{2}}}(2 \pi)^{4} \\
& \times \delta^{(4)}\left(q_{1}+q_{2}-\left(p_{1}+p_{2}\right)\right) \frac{q_{1}^{\alpha} q_{2}^{\beta}}{\left(p_{3}-q_{1}\right)^{2}-M_{W}^{2}} .
\end{aligned}
$$

Now, let us make some estimates. Consider the standard parametrization of $V_{\mathrm{CKM}}$. Suppose we choose the angles as $\theta_{1}=3.366, \theta_{2}=0.0307, \theta_{3}=0.0067$, and a phase $\delta_{\mathrm{CKM}}$ $=1.4633$ roughly consistent with experimentally known bounds. We specify in part a Yukawa texture by choosing the rotation matrix

$$
U_{u}=\exp \left[i\left(\begin{array}{ccc}
1 & 0.5 & 0.9 \\
0.5 & 1 & 0.6 \\
0.9 & 0.6 & -2
\end{array}\right)\right]
$$

and suppose $U_{i}=W_{i}$. With a array of random (real) numbers of order 1 for the $\lambda^{a n b r}$, we find the effective $C P$ violating imaginary coefficient to be distributed as shown in Fig. 3

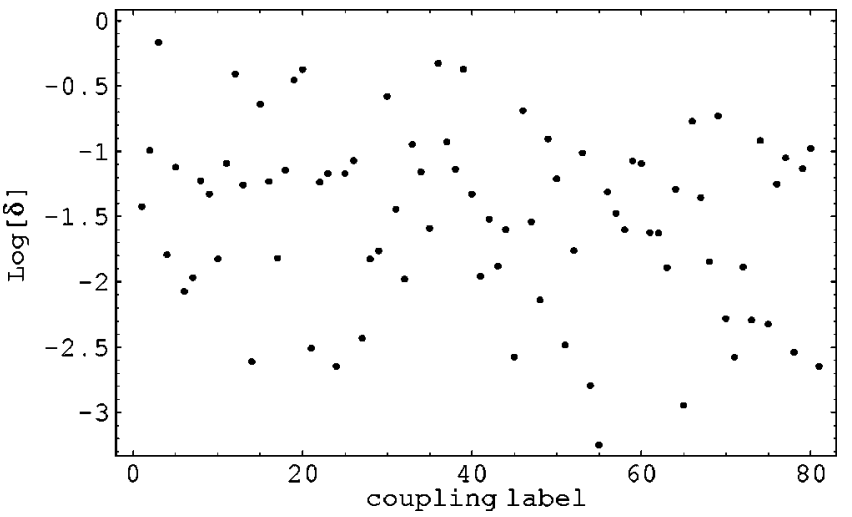

FIG. 3. A typical distribution of $C P$ violating coefficients from a random texture.

where we have summed over the indices $i, j$ as if the rest of the transition probability did not depend on $i$ and $j$. As expected, the quantity $\delta$ (analogous to the Jarlskog invariant in the presence of the dimension- 6 operators) is around 0.1 , much larger than the Jarlskog invariant of $\sim 10^{-5}$. We expect similar results if the $\lambda^{a n b r}$ are taken complex with random phases.

As for the $I_{s t i j}^{\alpha \beta}$ we have when the Mandelstam invariant $s \ll M_{W}^{2}$

$$
I_{s t i j}^{\alpha \beta} \sim 10 g^{\alpha \beta} \frac{1}{M_{W}^{2}}\left(s-\left(m_{u_{i}}+m_{d_{j}}\right)^{2}\right)
$$

while for the top quark,

$$
I_{s t i j}^{\alpha \beta} \sim 30 .
$$

Note that $M_{W}$ can be time dependent since

$$
M_{W} \approx \sqrt{2 \pi \alpha_{W}} v
$$

and $\alpha_{W}$ can be time dependent (anywhere between $0<\alpha_{W}$ $\leqslant 1)$. Although $v$ may be time dependent, we will take it to be approximately constant [25].

Now, let us write down the Boltzmann equation for our system of quarks and leptons. Denoting the phase space density of particles in an FRW cosmology with scale factor $a$ as $f_{x}$, we have

$$
\begin{aligned}
\frac{\partial f_{x}}{\partial t}- & \frac{\dot{a}}{a} \frac{|\vec{p}|^{2}}{E_{x}} \frac{\partial f_{x}}{\partial E_{x}} \\
= & \sum_{i, j, b, c, d} \int\left\{-f_{x} f_{b}\left|\mathcal{M}_{1}\right|^{2}-2 f_{x} f_{b} \operatorname{Re}\left\{\mathcal{M}_{1} \mathcal{M}_{2}^{*}\right\}\right. \\
& +\frac{f_{c} f_{d}}{f_{c}^{e q} f_{d}^{e q}} f_{x}^{e q} f_{b}^{e q}\left|\mathcal{M}_{1}\right|^{2}-2 \frac{f_{c} f_{d}}{f_{c}^{e q} f_{d}^{e q}} f_{x}^{e q} f_{b}^{e q} \operatorname{Re}\left\{\mathcal{M}_{1} \mathcal{M}_{2}^{*}\right\} \\
& -f_{x} f_{b}\left|\mathcal{M}_{3}\right|^{2}-2 f_{x} f_{b} \operatorname{Re}\left\{\mathcal{M}_{3} \mathcal{M}_{4}^{*}\right\} \\
& \left.+\frac{f_{i} f_{j}}{f_{i}^{e q} f_{j}^{e q}} f_{x}^{e q} f_{b}^{e q}\left|\mathcal{M}_{3}\right|^{2}-2 \frac{f_{i} f_{j}}{f_{i}^{e q} f_{j}^{e q}} f_{x}^{e q} f_{b}^{e q} \operatorname{Re}\left\{\mathcal{M}_{3} \mathcal{M}_{4}^{*}\right\}\right\} \\
& +\ldots
\end{aligned}
$$


where

$$
\int \equiv \frac{1}{2 E_{x}} \int \prod_{n \in \text { reactants }} d \Pi_{n}(2 \pi)^{4} \delta^{(4)}\left(p_{x}+\sum_{r \in \text { reactants }} p_{r}\right),
$$

is an operator generating an appropriate momentum integral (with the measure $d \Pi_{n}=d^{3} p_{n} /\left[2 E_{n}(2 \pi)^{3}\right]$ ), $\mathcal{M}_{1}$ is the amplitude for the tree level process $x b \rightarrow c d$, and $\mathcal{M}_{2}$ is associated with the one loop correction with intermediate states $i j$. The amplitude $\mathcal{M}_{3}$ is associated with the tree level amplitude of $x b \rightarrow i j$, where $i j$ are the intermediate states in the calculation of $\mathcal{M}_{2}$, and the one loop correction to $\mathcal{M}_{3}$ involving the intermediate states $c d$ (appearing in $\mathcal{M}_{1}$ ) is denoted as $\mathcal{M}_{4}$. Here, we have neglected the Pauli blocking factors for the Boltzmann collision integral. Although this is easily generalizable to include the Pauli blocking factors and although it is not justified when calculating an accurate number density evolution, the neglect is justified when calculating an upper bound on the baryon numbers produced. Hence, for clarity of physics we shall drop them in this paper. Because only the amplitudes with the fermion propagators cut in $\mathcal{M}_{2}$ and $\mathcal{M}_{4}$ contribute, one can show within the collision integral sum that $\operatorname{Re}\left\{\mathcal{M}_{1} \mathcal{M}_{2}^{*}\right\}=-\operatorname{Re}\left\{\mathcal{M}_{3} \mathcal{M}_{4}^{*}\right\}$. Note that the collision integral vanishes in equilibrium. (A related work on this kind of Boltzmann equation may be found in Ref. [26].)

Thus one arrives at the baryon number density evolution equation

$$
\begin{aligned}
\frac{1}{a^{3}} \frac{d\left(n_{B} a^{3}\right)}{d t}= & 2 \int_{a, b, i, j, c, d}^{\prime} b_{a} f_{a}^{e q} f_{b}^{e q} \\
& \times \operatorname{Re}\left\{\mathcal{M}_{1} \mathcal{M}_{2}^{*}(a b \rightarrow i j \rightarrow c d)\right\} \\
& \times\left[\frac{f_{i} f_{j}+f_{\bar{i}} f_{\bar{j}}}{f_{i}^{e q} f_{j}^{e q}}-\frac{f_{\bar{c}} f_{\bar{d}}+f_{c} f_{d}}{f_{c}^{e q} f_{d}^{e q}}\right]
\end{aligned}
$$

where $b_{a}$ is the baryon number associated with reactant $a$ and the prime on the integral denotes that all the momenta are to be integrated over. Note that for each baryon number associated with reactant $a$ to contribute to the baryon asymmetry, the intermediate and the final state must be out of equilibrium and not the initial reactant. This is expected from unitarity and $C P T$ which implies that [27]

$$
\sum_{j}|\mathcal{M}(i \rightarrow j)|^{2}=\sum_{j}|\mathcal{M}(\bar{i} \rightarrow j)|^{2}
$$

where $j$ sums over all final states: when the probability is summed over all final states, there cannot be any $C P$-violating enhancement or subtraction of the probability that survives. Also, note that although naively one might think that the right-hand side (RHS) of Eq. (27) would vanish, since the quantity inside the square bracket is odd under the exchange $i j \leftrightarrow c d$ and the summation is over all $i, j, c, d$, there is no cancellation because $\operatorname{Re}\left\{\mathcal{M}_{1} \mathcal{M}_{2}^{*}(a b \rightarrow i j\right.$ $\rightarrow c d)\}$ is also odd under the exchange $i j \leftrightarrow c d$. Finally, note that although we have only written down the scattering processes explicitly, the decay and inverse decay reactions have an analogous form.

Now, instead of accounting for the possible contributions to the baryon asymmetry from all the reactants together, let us put bounds on the maximum contribution that arises from each of the reactants. As explained in Appendix C, we can derive from Eq. (27)

$$
n_{B} \leqslant \frac{2 \eta}{a^{3}} \int d t \Gamma_{\mathrm{BV}}(t) a^{3} n_{f}
$$

where

$$
\eta \equiv \alpha_{W} \sum_{i j} I \operatorname{Im}\left\{\lambda^{a j b i} \lambda^{* a t b s} V_{i s} V_{t j}^{*}\right\} \sim 0.02 \frac{\bar{T}^{2}}{v^{2}}
$$

$\bar{T}^{2} \approx T_{\text {init }}^{2}+m_{\text {init }}^{2}-\left(m_{u i}+m_{d j}\right)^{2}, T_{\text {init }}$ and $m_{\text {init }}$ are temperature and mass scales, respectively, of the initial state (or final state) reactants, $m_{u i}$ and $m_{d j}$ are the masses of the particles in the loop, and $\Gamma_{\mathrm{BV}}$ is the tree-level baryon number violating reaction rate which can be read off from Table I. In the table the function $W$ defined as

$$
\begin{aligned}
W\left(m_{Q_{u}^{a}}, T\right) & \equiv \frac{m_{Q_{u}^{a}}^{2} T}{2 \pi^{2} n_{Q_{u}^{a}}^{e q}} K_{1}\left(m_{Q_{u}^{a}} / T\right) \\
& \approx \frac{1}{\sqrt{1+4\left(T / m_{\left.Q_{u}^{a}\right)^{2}}^{2}\right.}}
\end{aligned}
$$

is a time dilation function arising from the thermal averaging and $F\left(m_{Q}, m_{L}, T_{Q}, T_{L}\right)$ is a function of order 1 also arising from thermal averaging ( $K_{1}$ is a modified Bessel function of the second kind). Using these and other results presented in Appendix $\mathrm{C}$, we can place an upper bound on the baryon asymmetry generated by any single species out of equilibrium as

$$
\frac{n_{B}}{s}<2 \eta\left[\frac{\Gamma_{\mathrm{BV}}(t)}{\Gamma_{\mathrm{tot}}(t)}\right]_{\max }
$$

where the maximum is taken at any time during baryogenesis. Note that this bound is independent of cosmology (without specifying the time dependence of the scale factor a) as long as the usual Boltzmann equations are valid.

Let us first consider the quarks. Instead of writing $\Gamma_{\text {tot }}$ as the sum of rates for all the channels, it is more illuminating to consider separately the cases when $\Gamma_{\text {tot }}$ is dominated by either the baryon-number-conserving annihilation rate or the decay rate. The $B$-conserving annihilation rate is dominated by a $t$-channel quark annihilation into gluons, with the possible exception of the top quark for which the rate can be dominated by a weak scattering. Explicitly, we use 
TABLE I. The dominant reaction rates. The upper indices on leptons $L$ and quarks $Q$ label the family number while the subscript indices label denotes up-type or down-type (e.g., $L_{u}$ is a neutrino). All other quantum numbers are implicit. The small number $\epsilon$ indicates the splitting due to the $C P$-violating phases. Only the quark decay rates are displayed, as they will be the most relevant quantities. The coupling $\lambda$ of the dimension- 6 operator is assumed to be of order 1 .

\begin{tabular}{|c|c|c|c|}
\hline$\Gamma_{\mathrm{BV}}$ & Reaction & Magnitude & $\Delta(B+L)$ \\
\hline$\Gamma_{\mathrm{BV} \operatorname{dec}}(1+\epsilon)$ & $Q_{u}^{a} \rightarrow \bar{L}_{d}^{b} \bar{Q}_{d}^{s} \bar{Q}_{u}^{t}$ & $\lambda^{2}\left(\frac{m_{Q_{u}^{a}}}{M_{f}}\right)^{4} \frac{m_{Q_{u}^{a}}}{(2 \pi)^{3} 32}(1+\epsilon) W\left(m_{Q_{u}^{a}}, T_{Q}\right)$ & -2 \\
\hline$\Gamma_{\mathrm{BV} \mathrm{dec}}(1-\epsilon)$ & $\bar{Q}_{u}^{a} \rightarrow L_{d}^{b} Q_{d}^{s} Q_{u}^{t}$ & $\lambda^{2}\left(\frac{m_{Q_{u}^{a}}}{M_{f}}\right)^{4} \frac{m_{Q_{u}^{a}}}{(2 \pi)^{3} 32}(1-\epsilon) W\left(m_{Q_{u}^{a}}, T_{Q}\right)$ & 2 \\
\hline$\Gamma_{\mathrm{BV} \text { scatt }}(1+\epsilon)$ & $L_{d}^{b} Q_{u}^{a} \rightarrow \bar{Q}_{d}^{s} \bar{Q}_{u}^{t}$ & 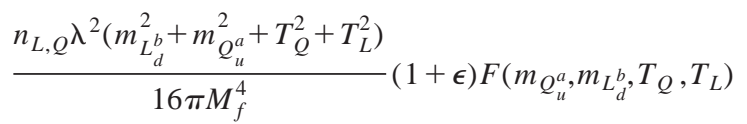 & -2 \\
\hline$\Gamma_{\text {BV scatt }}(1-\epsilon)$ & $\bar{L}_{d}^{b} \bar{Q}_{u}^{a} \rightarrow Q_{d}^{s} Q_{u}^{t}$ & 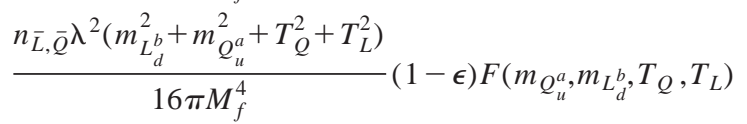 & 2 \\
\hline
\end{tabular}

$$
\begin{aligned}
\Gamma_{\mathrm{ann}} & \approx n_{Q} \frac{\alpha_{S}^{2}}{m_{Q}^{2}+T_{Q}^{2}} F_{2}\left(m_{Q}, T_{Q}\right) \\
\Gamma_{t-\mathrm{ann}} & \approx \Gamma_{\mathrm{ann}}\left(\frac{m_{t}}{M_{W}}\right)^{4}\left(\frac{\alpha_{W}}{\alpha_{S}}\right)^{2}\left|V_{\mathrm{CKM}}\right|^{2} \equiv \Gamma_{\mathrm{ann}} / E_{t}
\end{aligned}
$$

where $\left|V_{\mathrm{CKM}}\right|^{2}$ is the appropriate quark mixing matrix element squared, and $F_{2}$ is a function encoding the effect of thermal averaging, similar to $F$. The $B$-conserving decay rate is dominated by a weak decay rate, which is enhanced for the top quark due to the longitudinal $W$ component going on shell. We use

$$
\begin{aligned}
\Gamma_{\text {weak dec }} & \approx \frac{\alpha_{W}^{2}}{16 \pi}\left(\frac{m_{Q}}{M_{W}}\right)^{4} m_{Q}\left|V_{\mathrm{CKM}}\right|^{2} W\left(m_{\left.Q_{u}^{a}, T_{Q}\right)}\right. \\
\Gamma_{t-\text { dec }} & \approx \Gamma_{\text {weak dec }} \frac{\pi}{\alpha_{W}}\left(\frac{M_{W}}{m_{t}}\right)^{2} \equiv \Gamma_{\text {weak dec }} / F_{t} .
\end{aligned}
$$

Explicitly, $\quad E_{t} \approx\left(4 /\left|V_{\mathrm{CKM}}\right|^{2}\right)(v / 180 \mathrm{GeV})^{4}$ while $F_{t}$ $\approx 0.05(v / 180 \mathrm{GeV})^{-2}$, which implies that the weak decay rate is not much enhanced for the top quark through the Goldstone emission. Given these rates, we list the leading bound $n_{B} / s$ (i.e., the upper bound on the baryon fraction relevant to the case that the given reactions are the dominant contributions) in Table II. The lepton sector is similar except with $\alpha_{s} \rightarrow \alpha_{e m}$ and no top quark enhancements. In addition, the leading number density changing channel for the neutrinos may be coannihilations instead of self-annihilations. From the last section, we have $M_{f}>100 \mathrm{TeV}$ which implies

$$
\eta\left(M_{W} / M_{f}\right)^{4}<3 \times 10^{-13}\left(\frac{v}{180 \mathrm{GeV}}\right)^{2} .
$$

Finally, we need to constrain the number density of the particles. Since the elastic scattering rates are at least as large as the total inelastic scattering rates, to absolutely forbid any phase transitions, we restrict the number density of particles to be smaller than the thermal density at $T_{*}=30 \mathrm{GeV}$, i.e.,

TABLE II. $n_{B} / s$ for quarks. For the top quark, each of these ratios must be multiplied by $E_{t}$ or $F_{t}$. However, these extra factors do not change our conclusions.

\begin{tabular}{lcc}
\hline \hline Ratio no. & $\Gamma_{\mathrm{BV}} / \Gamma_{\mathrm{tot}}$ & $\left(n_{B} / s\right)_{\max }$ \\
\hline 1 & $\Gamma_{\mathrm{BV} \mathrm{dec}} / \Gamma_{\mathrm{ann}}$ & $\frac{2 \eta}{\alpha_{S}^{2}} \frac{m_{Q}^{4}}{M_{f}^{4}}\left[\frac{m_{Q}\left(m_{Q}^{2}+T_{Q}^{2}\right) W\left(m_{Q}, T_{Q}\right)}{n_{Q} F_{2}\left(m_{Q}, T_{Q}\right)}\right]_{\max }\left(\times E_{t}\right)$ \\
2 & $\Gamma_{\mathrm{BV} \mathrm{scatt}} / \Gamma_{\mathrm{ann}}$ & $\frac{\eta}{8 \pi \alpha_{S}^{2}} \frac{\left(m_{Q}^{2}+T_{Q}^{2}\right)\left(m_{f_{2}}^{2}+m_{Q}^{2}+T_{Q}^{2}\right)}{M_{f}^{4}}\left[\frac{n_{f_{2}}(t) F\left(m_{Q}, m_{f_{2}}, T_{Q}\right)}{n_{Q}(t) F_{2}\left(m_{Q}, T_{Q}\right)}\right]_{\max }\left(\times E_{t}\right)$ \\
4 & $\Gamma_{\mathrm{BV} \mathrm{dec}} / \Gamma_{\text {weak dec }}$ & $\frac{32 \pi \eta}{g^{4}}\left(\frac{M_{W}}{M_{f}}\right)^{4} \frac{\left(m_{f_{2}}^{2}+m_{Q}^{2}+T_{Q}^{2}+T_{f_{2}}^{2}\right)}{m_{Q}^{2}\left|V_{\mathrm{CKM}}\right|^{2}}\left[\frac{n_{f_{2}} F\left(m_{Q}, m_{f_{2}}, T_{Q}, T_{f_{2}}\right)}{m_{Q}^{3} W\left(m_{Q}, T_{Q}\right)}\right]_{\text {max }}\left(\times F_{t}\right)$ \\
\hline
\end{tabular}




$$
n_{Q, L}^{\max }<0.2 T_{*}^{3}
$$

if relativistic or

$$
n_{Q, L}^{\max }<0.13\left(m_{Q, L} T_{*}\right)^{3 / 2} e^{-m_{Q, L} / T_{*}}
$$

if nonrelativistic [28].

Now, consider the case when the branching ratio is approximated by ratio 3 in Table II. For the top quarks out of equilibrium, we have

$$
\text { ratio } 3=5 \times 10^{-15}\left(\frac{v}{180 \mathrm{GeV}}\right)^{2}
$$

while for all other reactants, we have

$$
\text { ratio } 3<6 \times 10^{-17}\left(\frac{1}{\left|V_{\mathrm{CKM}}\right|^{2}}\right)\left(\frac{v}{180 \mathrm{GeV}}\right)^{2}
$$

where we have used the bottom quark mass of about 4.3 $\mathrm{GeV}$. For the top quark, because the upper bound here is smaller than what is needed for sufficient baryogenesis and since the same numerator appears in ratio 1 , the $B$-violating decay channel is ruled out for the top quark. For the bottom quark, since the leading decay channel has $\left|V_{c b}\right|^{2}$ $\approx 1.6 \times 10^{-3}$, Eq. (41) also rules out sufficient baryogenesis. All other channels also fail the tests under ratios 1 and 3 which means that the $B$-violating decay channel cannot help in generating sufficient baryon asymmetry.

Consider now ratios 2 and 4 . Ratio 4 requires for the top quark that

$$
\frac{n_{B}}{s}<4 \times 10^{-15}
$$

if we assume that $F \sim W \sim 1$. Hence, ratios 3 and 4 completely rule out the top quark as a significant contributor to the baryon asymmetry.

Let us now consider the scattering of the out-ofequilibrium particle $f$ with every other particle labeled as $f_{2}$ in view of ratio 2 . It can easily be seen that ratio 2 is not very constraining, as it can in principle be made arbitrarily large. Hence, we can consider a more stringent version of the entropy bound corresponding to ratio 2, Eq. (29), which we rewrite as

$$
\begin{aligned}
\frac{n_{B}}{s} \leqslant & \frac{2 \eta\left(m_{f}^{2}+m_{f_{2}}^{2}+\bar{T}_{f}^{2}+\bar{T}_{f_{2}}^{2}\right)}{M_{f}^{4} s} \frac{1}{a^{3}} \\
& \times \int_{t_{B}}^{{ }^{t_{f}} d t a^{3} g_{1}\left(m_{f}, m_{f_{2}}, T_{f}, T_{f_{2}}\right) n_{f} n_{f_{2}}}
\end{aligned}
$$

where $s$ is the entropy density, $T_{i}$ is the temperature of particle species $i, \bar{T}_{i}$ is the characteristic temperature of species $i$ inside the integral, $g_{1}$ is a kinematic function of order $1, t_{B}$ marks the beginning time of baryogenesis, and $t_{f}$ marks the end time of baryogenesis. Instead of using the maximum contribution to the entropy, it is sufficient to consider any one contribution since we are placing an upper bound. Since we are guaranteed that the reaction changing the number density of $f$ is out of equilibrium, and there exists a contribution to the entropy from the weak interaction $\left(f f_{2}\right.$ $\rightarrow$ final products) with the matching integral of the form $\int_{t_{B}}^{t_{f}} d t a^{3} n_{f} n_{f_{2}}$ (since $f$ is necessarily out of equilibrium), we can write using Eq. (C9)

$$
\begin{aligned}
s \geqslant & \frac{\alpha_{W}^{2}\left|V_{\mathrm{CKM}}\right|^{2}\left(m_{f}^{2}+m_{f_{2}}^{2}+\bar{T}_{f}^{2}+\bar{T}_{f_{2}}^{2}\right)}{M_{W}^{4}} \frac{1}{a^{3}} \\
& \times \int_{t_{B}}^{{ }^{t_{f}} d t a^{3} g_{2}\left(m_{f}, m_{f_{2}}, T_{f}, T_{f_{2}}\right) n_{f} n_{f_{2}}}
\end{aligned}
$$

where $g_{2}$ is another order 1 dimensionless function. It is important to note that we have assumed the kinematic viability of the weak interaction in Eq. (44). Combining Eq. (44) and Eq. (43), we have

$$
\begin{aligned}
\frac{n_{B}}{s} & \leqslant \frac{2 \eta}{\alpha_{W}^{2}\left|V_{\mathrm{CKM}}\right|^{2}}\left(\frac{M_{W}}{M_{f}}\right)^{4} \\
& \approx 5 \times 10^{-13} \frac{1}{\left|V_{\mathrm{CKM}}\right|^{2}}\left(\frac{v}{180 \mathrm{GeV}}\right)^{2}
\end{aligned}
$$

independently of $\alpha_{W}$. Hence, with $\left|V_{\mathrm{CKM}}\right|^{2} \leqslant 10^{-3}$, sufficient baryon asymmetry generation seems feasible. A case in which Eq. (45) applies is when out of equilibrium $b$ quarks are interacting with the $c$ quarks at an effective temperature of $30 \mathrm{GeV}$. In particular, if the baryon number conserving weak interaction channel is dominated by $b c \rightarrow c s$ which gives the $\left|V_{c b}\right|^{2} \approx 1.6 \times 10^{-3}$ suppression, sufficient baryogenesis in this scenario seems possible. We will return to this possibility later and constrain it further.

Another possibility to evade Eq. (45) is to have the weak interaction be kinematically suppressed. In the case of an out of equilibrium $c$ quark, the reaction $c \nu_{\tau} \rightarrow s \tau$ has the kinematic suppression necessary to evade Eq. (45) in the limit of zero temperature since $m_{\tau} \approx 1.7 \mathrm{GeV}$ while $m_{c} \approx 1.5 \mathrm{GeV}$. In Table III, we list all the weak interactions that are kinematically suppressed such that sufficient baryogenesis is possible with respect to the constraints thus far.

According to Table III, some weak interactions are kinematically suppressed only below the temperature of the QCD phase transition, which is assumed to occur at around $T$ $\approx 0.2 \mathrm{GeV}[29]$. These interactions hence really are not suppressed in the temperature range of interest and the baryogenesis bound is not evaded for these reactants. Hence, these are labeled irrelevant in Table III. Only interactions involving $\nu_{\tau}$ then remain as viable candidates for the kinematically suppressed baryon number conserving channel, if we assume that its mixing with $\nu_{\mu}$ or $\nu_{e}$ is less than $10^{-4}$. If the neutrino sector is mixed, all the bounds return and there are no candidates that can generate sufficient baryon asymmetry [30].

In both cases where Eq. (45) allows sufficient baryogenesis, the baryon number-conserving weak interactions between the reactants contribute too little to the entropy to rule out baryogenesis. In such situations, let us consider whether 
TABLE III. The set of reactions evading the simplest bound. These reactions are suppressed when the temperature falls below the threshold temperature.

\begin{tabular}{ccccc}
\hline \hline $\begin{array}{l}\text { Out of } \\
\text { equilibrium } \\
\text { particle }\end{array}$ & $\begin{array}{c}\text { Weak } \\
\text { interactions }\end{array}$ & $\begin{array}{c}\text { Threshold } \\
\text { temp. } \\
(\mathrm{GeV})\end{array}$ & Relevant & E.g. BV \\
\hline$c$ & $c \bar{\nu}_{\tau} \rightarrow s \bar{\tau}$ & 0.4 & $\mathrm{Y}$ & $c \nu_{\tau} \rightarrow \bar{d} \bar{d}$ \\
$s$ & $s \nu_{\tau} \rightarrow u \tau$ & 1.5 & $\mathrm{Y}$ & $s \nu_{\tau} \rightarrow \bar{d} \bar{u}$ \\
$d$ & $d \nu_{\tau} \rightarrow u \tau$ & 1.7 & $\mathrm{Y}$ & $d \nu_{\tau} \rightarrow \bar{d} \bar{u}$ \\
$"$ & $d \nu_{\mu} \rightarrow u \mu$ & 0.1 & $\mathrm{~N}$ & \\
$u$ & $u \bar{\nu}_{\tau} \rightarrow d \bar{\tau}$ & 1.7 & $\mathrm{Y}$ & $u \nu_{\tau} \rightarrow \bar{d} \bar{d}$ \\
, & $u \bar{\nu}_{\mu} \rightarrow d \bar{\mu}$ & 0.1 & $\mathrm{~N}$ & \\
,$"$ & $u \bar{\nu}_{e} \rightarrow d \bar{e}$ & 0.006 & $\mathrm{~N}$ & \\
$e$ & $u e \rightarrow d \nu_{e}$ & 0.005 & $\mathrm{~N}$ & \\
$\nu_{\tau}$ & $\nu_{\tau} \bar{c} \rightarrow \bar{d} \tau$ & 0.21 & $\mathrm{~N}$ & \\
, & $\nu_{\tau} s \rightarrow u \tau$ & 1.505 & $\mathrm{Y}$ & $\nu_{\tau} s \rightarrow \bar{d} \bar{u}$ \\
, & $\nu_{\tau} d \rightarrow u \tau$ & 1.7 & $\mathrm{Y}$ & $\nu_{\tau} d \rightarrow \bar{d} \bar{u}$ \\
,, & $\nu_{\tau} \bar{u} \rightarrow \bar{d} \tau$ & 1.7 & $\mathrm{Y}$ & $\nu_{\tau} u \rightarrow \bar{d} \bar{d}$ \\
$\nu_{\mu}$ & $\nu_{\mu} d \rightarrow u \mu$ & 0.1 & $\mathrm{~N}$ & \\
,$"$ & $\nu_{\mu} \bar{u} \rightarrow \bar{d} \mu$ & 0.1 & $\mathrm{~N}$ & \\
$\nu_{e}$ & $\nu_{e} \bar{u} \rightarrow \bar{d} e$ & 0.005 & $\mathrm{~N}$ & \\
\hline \hline
\end{tabular}

the self-annihilation interaction contribution to the entropy is enough to dilute away the baryon asymmetry contribution. Start with Eq. (43) and again approximate $g_{1} \approx 1$. However, instead of Eq. (44), consider

$$
s \geqslant \frac{\left\langle\sigma_{A f_{2}} v\right\rangle}{a^{3}} \int_{t_{c}}^{t_{f}} d t a^{3} n_{f_{2}}^{2}+\frac{\left\langle\sigma_{A f} v\right\rangle}{a^{3}} \int_{t_{B}}^{t_{f}} d t a^{3} n_{f}^{2}
$$

which corresponds to the entropy bound arising from selfannihilation reaction contributions, where the contribution to the entropy from the species $f_{2}$ only begins once it goes out of equilibrium, at time $t_{c}$, while the $f$ self-annihilation contribution persists throughout the entire duration $\left[t_{B}, t_{f}\right]$ of baryogenesis. Here, the thermal averaged quantities (such as $\left.\left\langle\sigma_{A f} v\right\rangle\right)$ have their temperature fixed at some value, characteristic of the integration interval. The advantage of considering the bound arising from self-annihilation reactions is that they change the number density and are always possible at tree level if there is a neutrino lighter than (or degenerate with) $\nu_{\tau}$. We shall assume that the self-annihilation channel is open $[31,32]$. Suppose first that the $n_{f_{2}}^{2}$ term dominates over the second term. Then, using the elementary inequality

$$
n_{f_{2}}^{2}+n_{f}^{2} \geqslant 2 n_{f} n_{f_{2}}
$$

we can write

$$
\frac{n_{B}}{s} \leqslant \frac{R}{2 M_{f}^{2}\left\langle\sigma_{A f_{2}} v\right\rangle}\left[1+\frac{\left\langle\sigma_{A_{f_{2}}} v\right\rangle}{\left\langle\sigma_{A f} v\right\rangle}+\xi\right]
$$

where we have defined

$$
R \equiv \frac{2 \eta\left(m_{f}^{2}+m_{f_{2}}^{2}+\bar{T}_{f}^{2}+\bar{T}_{f_{2}}^{2}\right)}{M_{f}^{2}}
$$

and

$$
\xi \equiv \frac{\int_{t_{B}}^{t_{c}} d t a^{3} n_{f_{2}}^{2}}{\int_{t_{c}}^{t_{f}} d t a^{3} n_{f_{2}}^{2}}
$$

which measures how much the $f_{2}$ particles can contribute to baryogenesis through interaction with out-of-equilibrium particle $f$ without contributing to the entropy. This can be seen from noting that $n_{f_{2}}$ is the equilibrium distribution during $t \in\left[t_{B}, t_{c}\right]$ by definition. Producing baryon asymmetry for a long time without producing any entropy (i.e. letting $t_{c}$ approach $t_{f}$ ) is the best that can be done to circumvent the suppression of the dimension 6 operator. For sufficient baryogenesis $\left(n_{B} / s \simeq 10^{-10}\right)$, we thus require

$$
\xi \geqslant \frac{\left\langle\sigma_{A_{f_{2}}} v\right\rangle}{\left\langle\sigma_{A f} v\right\rangle}\left[\frac{2}{\delta}-1\right]-1
$$

where

$$
\delta \equiv \frac{R}{M_{f}^{2}\left\langle\sigma_{A f} v\right\rangle} \times 10^{10}
$$

If the $n_{f}^{2}$ dominates in Eq. (46), one can similarly derive Eq. (51), thereby showing that it is true in general. Now, noting that one can write

$$
\int_{t_{B}}^{t_{f}} d t a^{3} n_{f}^{2} \leqslant \delta \int_{t_{B}}^{t_{f}} d t a^{3} n_{f} n_{f_{2}}
$$

and using Eq. (47), one can write

$$
(2-\delta) \int_{t_{B}}^{t_{f}} d t a^{3} n_{f} n_{f_{2}} \leqslant \int_{t_{B}}^{t_{f}} d t a^{3} n_{f_{2}}^{2} .
$$

It follows that

$$
\int_{t_{B}}^{t_{f}} d t a^{3} n_{f} n_{f_{2}} \leqslant \frac{\left(1+\frac{1}{\xi}\right)}{(2-\delta)} \int_{t_{B}}^{t_{c}} d t a^{3} n_{f_{2}}^{2}
$$

which is useful if $\xi>1$ and $\delta<1$, since as we mentioned before, $n_{f_{2}}$ is the equilibrium distribution during the time interval $\left[t_{B}, t_{c}\right]$. Hence, we shall use Eq. (55) with parametrized cosmology to put a bound on cosmology from the condition of sufficient baryogenesis.

We parametrize the unknown cosmology as

$$
a \propto t^{n},
$$

$$
T \propto a^{-\nu}
$$


and determine $t_{c}$ by the out-of-equilibrium condition

$$
\frac{\dot{a}\left(t_{c}\right)}{a\left(t_{c}\right)}=\max \left[j\left\langle\sigma_{A f_{2}} v\right\rangle n_{f_{2}}\left(t_{c}\right), \Gamma_{f_{2}}\right]
$$

where $j$ is an $\mathcal{O}(1)$ constant and $\Gamma_{f_{2}}$ is the decay rate of $f_{2}$. Because the ratio of the decay rate $\Gamma_{f_{2}}$ to the expansion rate increases as a function of time, when the decay becomes the dominant chemical potential changing reaction, it can lead to an out-of-equilibrium number density of the $f_{2}$ only if the final states of the decay are not in equilibrium. Hence, the maximum above applies only if the decay products are out of equilibrium, and otherwise, only the self-annihilation should be taken into account. Furthermore, note that any choice of $t_{c}$ that we make in Eq. (58) corresponds to a different choice of cosmology because $t_{c}$ sets the scale of expansion rate of the universe. The larger $t_{c}$ is, the slower is the expansion rate, and hence the slower the dilution rate of any accumulation of baryon asymmetry. As far as interpreting the parameter $\nu$ is concerned, it is shown in Appendix D that $\nu \leqslant 1$ for most physical systems (even with external sources such as the inflaton) confined to 3 spatial dimensions, unless there exists a cooling mechanism different from the expansion of the 3 spatial dimensions. However, with the presence of extra dimensions, the energy exchange with the bulk exotics may induce a more rapid cooling allowing for $\nu>1$. If $\left\langle\sigma_{A f_{2}} v\right\rangle n_{f_{2}}\left(t_{c}\right)$ determines the out of equilibrium time of $f_{2}$, we find

$$
\begin{aligned}
\frac{n_{B}}{s}<\frac{\tilde{c}_{2} R}{j \tilde{c} M_{f}^{2}\left\langle\sigma_{A f_{2}} v\right\rangle} \frac{m_{f_{2}}^{2}}{T_{c}^{2}} \frac{\left(1+\frac{1}{\xi}\right)}{(2-\delta)} \frac{1}{\nu \pi^{2}} \\
\quad \times \int_{T_{c} / T_{B}}^{1} d x x^{(1 / \nu)(1 / n+3)-3} \frac{K_{2}^{2}\left(\frac{m_{f_{2}}}{T_{c}} x\right)}{K_{2}\left(\frac{m_{f_{2}}}{T_{c}}\right)}
\end{aligned}
$$

where $\tilde{c} \equiv 2 \pi^{2} g_{* S} / 45, g_{* S}$ is the number of degrees of freedom contributing to the entropy at time $t_{c}$, and the factor of $\tilde{c}_{2}$ accounts for (the square of) the degrees of freedom in the $f_{2}$ number density ( $K_{2}$ is a modified Bessel function of the second kind). We will assume that $g_{* S}$ accounts for at least the 16 degrees of freedom of the gluons in the thermal bath. As for $\tilde{c}_{2}$, if $f_{2}$ for example is a quark, then it will be 9 for the 3 color degrees of freedom (the 2 helicity states were already accounted for, which means that if $f_{2}$ were Weyl neutrinos, this should be divided by 4). If $\Gamma_{f_{2}}$ determines the out-of-equilibrium time of $f_{2}$, then this quantity should be multiplied by

$$
\frac{\left\langle\sigma_{A f_{2}} v\right\rangle m_{f_{2}}^{2} T K_{2}\left(\frac{m_{f_{2}}}{T}\right)}{\pi^{2} \Gamma_{f_{2}}} .
$$

For the relevant reactions in Table III, a $\nu_{\tau}$ and a quark are always paired. For the quarks out of equilibrium, $m_{f_{2}}$ $=m_{\nu_{\tau}} \approx 0$ and $m_{f}=m_{q}$ :

$$
\begin{aligned}
& \xi \geqslant \frac{\alpha_{W}^{2}\left(\bar{T}_{\nu_{\tau}}^{2}+m_{\nu_{\tau}}^{2}\right)\left(m_{q}^{2}+\bar{T}_{q}^{2}\right)}{\alpha_{s}^{2} M_{Z}^{4}}\left[\frac{2}{\delta}-1\right]-1, \\
& \delta=\frac{2 \eta\left(m_{q}^{2}+m_{\nu_{\tau}}^{2}+\bar{T}_{q}^{2}+\bar{T}_{\nu_{\tau}}^{2}\right)}{M_{f}^{2}} \frac{\left(m_{q}^{2}+\bar{T}_{q}^{2}\right)}{\alpha_{s}^{2} M_{f}^{2}} \times 10^{10} .
\end{aligned}
$$

These equations tell us that for all effective temperatures (between 1.7 and $0.2 \mathrm{GeV}$ ) and quark masses (less than $2 \mathrm{GeV}$ ) relevant to our scenario, we have the conditions $\delta$ $\ll 1$ and $\xi \gg 1$, which allow nontrivial bounds to come from Eq. (59). From Eq. (59), it is easy to show that there are no values of $\nu>0$ and $n>0$ such that sufficient baryon asymmetry is generated. Hence, there are no scenarios in which there is sufficient baryogenesis through out-of-equilibrium quarks interacting with $\nu_{\tau}$.

For the case of $\nu_{\tau}$ out of equilibrium and $q$ in equilibrium, $m_{f_{2}}=m_{q}$ and $m_{f}=m_{\nu} \approx 0$ and we have a similar result:

$$
\begin{aligned}
\xi \geqslant & \frac{\alpha_{s}^{2} M_{z}^{4}}{\alpha_{W}^{2}\left(\bar{T}_{q}^{2}+m_{q}^{2}\right)\left(m_{\nu_{\tau}}^{2}+\bar{T}_{\nu_{\tau}}^{2}\right)}\left[\frac{2}{\delta}-1\right]-1 \\
\delta= & \frac{2 \eta\left(m_{q}^{2}+m_{\nu_{\tau}}^{2}+\bar{T}_{q}^{2}+\bar{T}_{\nu_{\tau}}^{2}\right)}{M_{f}^{2}} \frac{M_{z}^{4}}{\alpha_{W}^{2}\left(m_{\nu_{\tau}}^{2}+\bar{T}_{\nu_{\tau}}^{2}\right) M_{f}^{2}} \\
& \times 10^{10} .
\end{aligned}
$$

Again, it is easy to check that for all relevant effective temperatures (between 1.7 and $0.2 \mathrm{GeV}$ ) and quark masses (less than $2 \mathrm{GeV}$ ), we have the conditions $\delta \ll 1$ and $\xi \gg 1$ which allow nontrivial bounds to come from Eq. (59). As before, Eq. (59) rules out the possibility of sufficient baryogenesis for $n>0$ and $\nu>0$. Hence, even neglecting the mixings, it seems nearly impossible to have sufficient baryogenesis with $\nu_{\tau}$ through the dimension-6 operator.

Let us now return to the case where the baryon number conserving weak interaction channel is dominated by $b c$ $\rightarrow c s$ which was seen to have sufficient suppression for us to further consider the possibility of successful baryogenesis. In applying the reasoning that led to Eq. (59), we can improve the bounds compared to just Eq. (59) by considering that the entropy also has a lower bound at temperature $T_{c}$ of

$$
s\left(T_{c}\right) a^{3}\left(T_{c}\right)>n_{g}\left(t_{B}\right) a^{3}\left(t_{B}\right)+n_{f 2}\left(t_{B}\right) a^{3}\left(t_{B}\right)
$$

where $n_{g}$ corresponds to the density of gluons and by putting $R$ inside the time integral and tying all the temperature scales together. The bound then becomes (similar to before) the smaller of either 


$$
\begin{aligned}
\frac{n_{B}}{s}< & \frac{\tilde{c}_{2}}{\nu M_{f}^{2}\left\langle\sigma_{A f_{2}} v\right\rangle} \frac{m_{f_{2}}^{2}}{M_{f}^{2}}\left(\frac{T_{c}}{T_{B}}\right)^{-3 / \nu} \\
& \times \int_{T_{c} / T_{B}}^{1} d x x^{(1 / \nu)(1 / n+3)-3} \\
& \times \frac{\left(m_{f}^{2}+m_{f_{2}}^{2}+2 T_{c}^{2} / x^{2}\right) T_{c} \eta(x) K_{2}^{2}\left(\frac{m_{f_{2}}}{T_{c}} x\right)}{K_{2}\left(\frac{m_{f_{2}}}{T_{c}}\right)\left(\sqrt{\tilde{c}} m_{f_{2}}^{2} T_{B} K_{2}\left(\frac{m_{f_{2}}}{T_{B}}\right)+\pi^{2} n_{g}\left[T_{B}\right]\right)}
\end{aligned}
$$

or Eq. (59). The large enhancement for the $b$-c interaction scenario in both Eq. (59) and Eq. (66) comes from the long time $t_{c}$ during which baryogenesis occurs while $f_{2}$ is in equilibrium. This leads to an accumulation of any net gain in baryon asymmetry over the entropy. More explicitly, $t_{c}$ varies as $1 / K_{2}\left(m_{f_{2}} / T_{c}\right)$ [or the equivalent factor from Eq. (60) when appropriate] which comes from the exponentially large ratio of the short distance physics scale to the expansion rate scale when $f_{2}$ goes out of equilibrium. The expansion rate must be exponentially small when $f_{2}$ goes out of equilibrium (if at all) because the self-annihilation reaction must maintain equilibrium even in the nonrelativistic regime in which the number densities are exponentially suppressed. The resulting bound with $m_{f}=m_{b}=4.3 \mathrm{GeV}$ and $m_{f_{2}}=m_{c}$ $=1.5 \mathrm{GeV}$ is shown in Fig. 4. As one can see, even with such an enhancement, there is insufficient baryogenesis (or marginally enough) in most cases. Even for the marginal cases, we do not expect the scenario to be realizable given the conservative nature of the bound. Other scenarios with small $\left|V_{C K M}\right|^{2}$ weak interactions while the heavier particle is out-of-equilibrium (i.e., out-of-equilibrium $b$ interacting with $u$, as well as out-of-equilibrium $c$ interacting with $d$ ) fail to generate sufficient baryogenesis for similar reasons.

If we do not assume thermal equilibrium initial conditions (before relevant species go out-of-equilibrium), then as before we may consider the $b$ being in equilibrium while the $c$ quark is out of equilibrium. According to our explanation of the enhancement for this scenario before, the larger the mass of the particle that stays in equilibrium until temperature drops to around $0.2 \mathrm{GeV}$, the smaller the exponentially suppressed density of nonrelativistic particles, and then as long as the self-annihilation determines the out-of-equilibrium time $t_{c}$, the longer is the time $t_{c}$ over which the baryon number is generated, leading to a larger accumulation of baryon asymmetry. Since $m_{b}>m_{c}$ in this scenario, we thus expect larger accumulation of baryon asymmetry over the entropy in this scenario. In fact, the self-annihilation reaction becomes small by the temperature $T=0.2 \mathrm{GeV}$ to the extent that it no longer controls the out-of-equilibrium time (instead it is determined by the $b$ decay). The competing entropy generation is suppressed because of $\left|V_{c b}\right|^{2} \sim 10^{-3}$ for the weak interactions, and other reactions generating entropy are insufficient as well in diluting the baryon asymmetry.

For completeness, one should note that since $m_{f}<m_{f_{2}}$ in

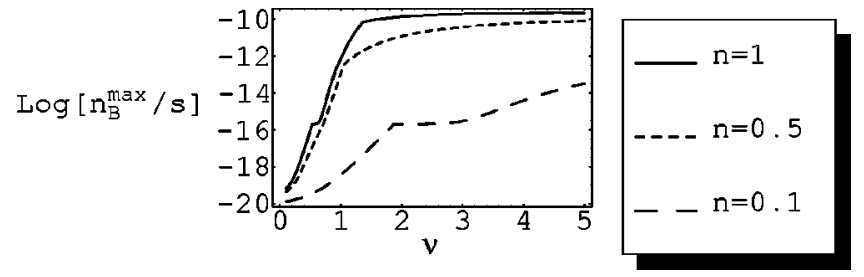

FIG. 4. A conservative upper bound for the baryon asymmetry generated by out of equilibrium $b$ quarks interacting with $c$ quarks. The parameters $n$ and $\nu$ parametrize various possible nonstandard cosmologies. A value $n>1$ corresponds to inflating cosmologies and in the absence of abnormal cooling $\nu \leqslant 1$. This scenario does not generate enough baryon asymmetry.

this scenario there is a contribution to the entropy not accounted for by Eq. (59) and Eq. (66). This comes from the fact that $b$ undergoes out-of-equilibrium decay to $c$ even though $b$ has a chemical equilibrium distribution. That is because $c$ by definition is out of equilibrium and thus cannot give the inverse decay reaction necessary for the $b$ decay to conserve entropy. However, we can check explicitly this contribution to the entropy contribution is negligible, even though it is proportional to the integration time $t_{c}$, because of the smallness of the decay rate. Also, since the $c$ is out of equilibrium, the reaction of $b-\bar{b}$ annihilation into $c-\bar{c}$ will also contribute to the creation of new entropy. However, one can check that this contribution is also insufficient to dilute away the baryon asymmetry generated. The numerical bounds shown for this scenario in Fig. 5 indicate that this scenario is viable at the level of our analysis.

The most obvious difficulty with this scenario is that another mechanism is required to set the initial density of $b$ quarks to be much larger than that of $c$, as well as to maintain that hierarchy. For example, for temperatures far above $m_{c}$, the gluons which keep $b$ quarks in equilibrium will tend to produce a large number of $c$ quarks that will be close to chemical equilibrium. Since a high initial temperature is crucial, some mechanism besides just the expansion of the universe is required to keep $c$ out of equilibrium while $b$ is in equilibrium. Even if the $c$ densities could somehow be kept small, since the self-annihilation cross sections for $b$ and $c$ are approximately the same, if the cosmological expansion is responsible for the out-of-equilibrium condition, then it must delicately tune itself to be smaller than the $b$ scattering rate while larger than that of the $c$ 's. However, our "modelindependent" analysis does not rule out this scenario, and it may be viable in a complicated and fortuitous setting.

As for the scenario in which $b$ quarks are in equilibrium while $u$ quarks are out of equilibrium, it will be even more difficult (if not impossible) to keep $u$ quarks out of equilibrium for the required "exponentially long" duration $t_{c}$, because the gluons which are supposed to be in equilibrium have one of their strongest equilibration reactions through $u$ $\bar{u}$ creation [33]. For the scenario with other quarks (besides the top and the bottom) in equilibrium during baryogenesis, the exponential enhancement of the duration of baryogenesis is too weak to allow sufficient baryogenesis.

Hence, remarkably, only one scenario, namely out-ofequilibrium $c$ quarks interacting with equilibrium $b$ quarks, 


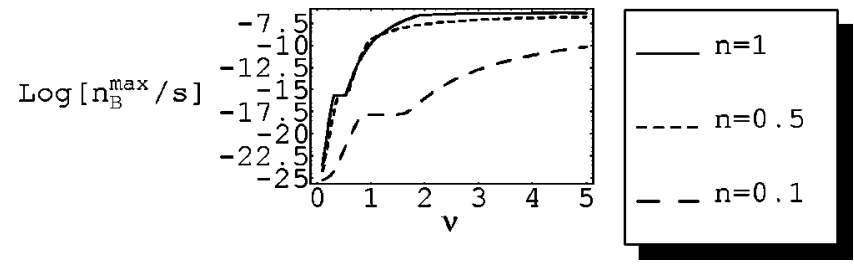

FIG. 5. A conservative upper bound for the baryon asymmetry generated by out-of-equilibrium $c$ quarks interacting with $b$ quarks. The parameters $n$ and $\nu$ are as before. For nonstandard cosmologies, sufficient baryogenesis may be possible. However, as explained in the text, the out-of-equilibrium condition seems difficult to maintain.

may generate sufficient baryon asymmetry with the dimension 6 operator if the out-of-equilibrium mechanism can be appropriately engineered. However, it would be surprising if such a realistic scenario can be constructed.

\section{CONCLUSION}

We have studied a new scenario of baryogenesis involving dimension-6 baryon-number-violating operators that would generically arise from integrating out ultraviolet degrees of freedom in the context of the standard model. The question to be answered in this scenario was whether a timedependent dimensionless suppression coefficient for the dimension- 6 operator can allow sufficient baryogenesis between the effective temperatures of $30 \mathrm{GeV}$ and $0.2 \mathrm{GeV}$, if the only fields that are allowed to participate in the short distance physics are those of the standard model. The upper bound on the temperature is motivated from the cosmological bound on $\mathrm{KK}$ graviton decay while the lower bound is purely for our calculational feasibility (related to the QCD phase transition). The time-dependent suppression coefficient was motivated from the generic possibility that with the Arkani-Hamed-Schmaltz mechanism of suppressing proton decay, the proton decay suppression factor can be time dependent.

Unfortunately, if the fundamental scale setting the size of the unsuppressed dimension- 6 operator is forced up to values $M_{f}>100 \mathrm{TeV}$ by the bound from neutron-antineutron oscillations, the branching ratio of $B$-violating interactions to $B$-conserving interactions is set by the quantity $\left(180 \mathrm{GeV} / M_{f}\right)^{4} \leqslant 10^{-13}$. We have shown this to be too small to allow sufficient baryogenesis for most cases. Another way to view this bound is that as the dimension- 6 operator creates baryon asymmetry, the same reactants participate in creating entropy through $B$-conserving operators. The competition between the two types of reactions is almost always dominated by the $B$-conserving one. Hence, within the perturbative setting where the 4D effective action is valid, sufficient baryogenesis in this scenario is in most cases impossible almost independently of cosmology.

There is one scenario which cannot simply be ruled out by the general analysis. This involves out-of-equilibrium $c$ quarks interacting with equilibrium $b$ quarks from a temperature of about $30 \mathrm{GeV}$ until a temperature of $0.2 \mathrm{GeV}$. The reason why this scenario evades the suppression is that the primary entropy generation channel is through the $b-\bar{b}$ selfannihilation into $c-\bar{c}$ instead of the $b-c$ weak interaction, since $\left|V_{c b}\right|^{2} \sim 10^{-3}$. Furthermore, given that nonstandard cosmology of braneworlds may give a slow expansion rate, one can have baryogenesis persist for a long time period, giving a large integrated pileup. However, given that $c$ quarks are produced efficiently both by the gluons that keep the $b$ quarks in equilibrium as well as the $b$ quarks which undergo decay, setting up the necessary hierarchy in densities to keep $c$ out-of-equilibrium while $b$ in equilibrium seems difficult. However, this scenario does pass the general tests not relying upon the specifics of the out-of-equilibrium mechanism.

Since neutrinos are almost massless and have a vanishingly small tree level annihilation cross section in the limit of small $\sqrt{s}$, one would naively think that this channel might be weak enough for the baryon-number-violating operator to compete with it. Unfortunately, this turns out not to be the case.

It is interesting to note that even with just the standard model fields, the presence of nonrenormalizable operators implies the existence of additional $C P$-violating rephasing invariants beyond the Jarlskog parameter. In any theory, the number of physical $C P$-violating phases is given by the number of couplings in the Lagrangian that are allowed to be complex, minus the dimension of the symmetry group which describes phase redefinitions of the fields: introducing new effective operators into the SM automatically creates more $C P$-violating invariants, even if all the couplings of the new operators are real in some basis. Hence, it is not the smallness of the Jarlskog invariant that prevents the success of our baryogenesis scenario. It is merely the fact that unless neutron-antineutron oscillations are suppressed by some means other than the fundamental scale, the phenomenologically acceptable scale of $100 \mathrm{TeV}$ is still too large for dimension-6 operators to play a significant role for baryogenesis below the temperature of the electroweak phase transition.

As with any "no go" arguments, there are many loopholes in our conclusion (besides the one remotely possible scenario that we already mentioned). First, we neglected any possible nonperturbative physics. The 4D coupling constants are inversely proportional to the volume of the extra dimensions in which the gauge fields propagate. Thus, if the volume of the extra dimensions was small at some epoch in the early Universe, then nonperturbative physics may dominate (see Appendix B), given that the perturbative contributions to $n_{B} / s$ are insufficient in our scenario. For example, the $S U(2)_{L}$ instanton effects may become unsuppressed, giving rise to a scenario similar to that of sphaleron transitions at the electroweak phase transition. Also, for effective temperatures below about $0.2 \mathrm{GeV}$, non-perturbative QCD effects will be relevant, since the quark degrees of freedom become confined. Although this is not likely to change the suppression of the dimension- 6 operator, the kinematics and matrix elements will be very different from our perturbative calculation done in the regime of deconfined quarks.

Secondly, we have neglected all effects of the KK modes; 
or, in the higher dimensional picture, we have neglected the fact that the space may be inhomogeneous in the higher dimensions. For example, one may envisage a scenario in which there is a phase transition which localizes the wave functions of the quarks and the leptons. A proper description of quark-lepton separation in higher dimension will require analysis beyond the zero mode. However, this type of effect will most likely play a role in enhancing the nonequilibrium condition rather than changing the baryon-number-violating branching ratio. Hence, our conclusion is most likely robust with respect to this assumption.

Thirdly, we have neglected all $C P T$ violation effects that must exist because of the time dependence of the quarklepton separation. If the time scale associated with the quarklepton separation process is very short, then there may be significant contributions from these effects to enhance the baryon number violating channels. However, such models will probably be severely constrained by the restriction on bulk graviton production, just as the reheating temperature is severely constrained.

Hence, although there are loopholes and caveats to our "no go" claims, it seems fair to conclude that with only perturbative physics, degrees of freedom beyond the standard model fields must play a significant role in baryogenesis if the effective temperature of the Universe never exceeds 30 $\mathrm{GeV}$ (which is a very conservative upper bound for the maximum temperature in models with large extra dimensions, if the fundamental scale is to be accessible to collider experiments). The requirement of beyond the standard model field content is not an obvious statement, considering that we hardly impose any restrictions on the cosmology and that there is an unsuppressed dimension-6 operator as well as an effectively large $C P$ violating phase available for baryon asymmetry generation. Of course, our "no go" claims would be significantly relaxed if the dimension-9 operator responsible for neutron-antineutron oscillations is suppressed by some symmetry mechanism which is introduced by hand to supplement the geometrical suppression of proton decay operators.

In closing, note that even if the fundamental scale can be lowered by evading the bound from neutron-antineutron oscillation, there is a significant challenge in this type of scenario as the quarks (and/or leptons) must be forced out of equilibrium at rather high temperatures. To accomplish this, it is possible to lower the Planck scale during baryogenesis by having a small extra dimensional volume during that period. However, there are severe restrictions coming from bulk graviton production in such cases. Furthermore, it is an extremely difficult challenge to find a time dependent potential for the scalar fields localizing the quarks and the leptons, such that the initial quark-lepton separation departs sufficiently from today's equilibrium value in a natural manner. We leave variations on our model that may generate sufficient baryon asymmetry to future studies.

\section{ACKNOWLEDGMENTS}

We thank Aaron Grant, Nima Arkani-Hamed, Misha Shaposhnikov, Lisa Everett, Rocky Kolb, Antonio Riotto,
Marty Einhorn, Gordy Kane, Antonio Masiero, Tony Gherghetta, Alexander Dolgov, Karim Benakli, Gianfrancesco Giudice and Rula Tabbash for helpful conversations and Boris Körs for correspondence. This research was supported in part by DOE Grant DE-FG02-95ER40899, Task G.

\section{APPENDIX A: ADEQUATE SUPPRESSION OF PROTON DECAY}

To find the minimum value of $L$ consistent with proton stability, we consider the decay process in the fourdimensional low energy theory, where we have argued that four-fermion operators give the largest contribution. (Processes that cannot be written as four-fermion effective operators, for example wormholes, are negligible for the value of $L$ that we find is needed.) The proton decay rate calculation for these operators is analogous to muon decay in the Fermi theory, giving an estimate of

$$
\tau_{p} \approx\left(\lambda v_{s} / M_{f}^{2}\right)^{-2} m_{p}^{-5}=\left(\lambda v_{s}\right)^{-2} a^{2} M_{f}^{4} m_{p}^{-5}
$$

for the "partial lifetime" of decay into any allowed channel, where $\lambda$ is a dimensionless constant of order 1. Modedependent experimental lower limits on the proton lifetime [4] require $\tau_{p}>2 \times 10^{31} \mathrm{yr}$ for some modes, which would translate into $M_{f}>3 \times 10^{15} \mathrm{GeV}$ in the absence of the suppression factor $v_{s}$. Thus

$$
a \leqslant\left(M_{f} \cdot 3 \times 10^{15} \mathrm{GeV}\right)^{2} \approx 10^{21-23} \approx e^{48-53}
$$

where the range quoted is for a fundamental scale ranging from 100 to $10 \mathrm{TeV}$. The conclusion is that about 50 $e$-foldings of suppression are required at the present epoch. This is easy to obtain if $v_{s} \sim e^{-\mu^{2} L^{2}}$ and $\mu$ is not too small relative to $M_{f}$, but is marginally consistent with perturbativity of $\mathrm{SU}(2)_{W}$, which begins to break down at $M_{f} R_{5}>30$ (recall that $R_{5} \geqslant L$ ), for proton decay mediated by states of mass $M_{f}$, for which $a \sim e^{-k M_{f} L}$ with $k$ of order 1 .

\section{APPENDIX B: OTHER POTENTIAL SOURCES OF B VIOLATION}

One should also consider nonperturbative processes which may affect $B$ violation and baryogenesis [34,35]. Even without the introduction of $B$-violating operators, the baryon number current is only conserved up to a $\mathrm{SU}(2)_{W}$ anomaly, and it is well known that topologically nontrivial gauge configurations can lead to a change in $B+L$, either by quantum tunneling via instantons, or thermal excitation via sphalerons [36]. The rates for $B$ violation by instantons and sphalerons also have exponential suppression factors $e^{-8 \pi / g_{2}^{2}}$ and $e^{-M_{\mathrm{S}} / T}$ respectively, where $M_{S}$ is the sphaleron energy given by $M_{\mathrm{S}} \approx 2 M_{W} / \alpha_{W}[37,38]$. If the total length of the fifth dimension $R_{5}$ were to change with time then, assuming a constant five-dimensional gauge coupling $g_{5}$, these processes could become important. Given the relationship $g_{4}^{2}=g_{5}^{2} / R_{5}$ for the weak gauge coupling, instantons would be exponentially larger for smaller $R_{5}$; the dependence is just $e^{- \text {const } \times R_{5}}$, 
so the instanton enhancement at small $R_{5}$ would shadow the increase in processes mediated by massive states. However, it can easily be checked that the instanton rate today is many orders of magnitude smaller than the bound on such perturbative processes, therefore instantons can never have dominated over the perturbative operators, unless the perturbative operators were further suppressed by many orders of magnitude (for example, if they vanished by an exact discrete symmetry). The sphaleron rate is more difficult to estimate, since in addition to the temperature dependence one would have to model how the Higgs vacuum expectation value (VEV) changes in response to changing $R_{5}$. On the simplest assumption of a constant VEV the sphaleron rate is actually independent of $R_{5}$ and, as in four dimensions, depends just on $T$. This is a counterexample to the claim that all processes are exponentially suppressed with increasing $R$; it is understood by noting that the formation of sphaleron configurations is a classical, thermally-activated process, in contrast to quantum effects which are suppressed by a massive propagator or by the Euclidean action. Unsuppressed sphaleron transitions "wash out" any baryon asymmetry generated at temperatures above the electroweak scale [39]; on the assumption of a very low maximum temperature of the Universe, which is required in low-scale models, sphaleron effects will be completely negligible.

\section{APPENDIX C: BOLTZMANN EQUATIONS AND ENTROPY}

Consider first the entropy of one species of particles. Divide the phase space of particles into cells and let the cells be numbered $i=1,2,3, \ldots$ where each cell contains $N_{i}$ identical particles. Let $G_{i}$ be the phase space volume that can be occupied by one particle in the cell $i$. The total phase space of the $i$ th cell is

$$
\Delta \Gamma_{i}=\frac{G_{i}^{N_{i}}}{N_{i} !}
$$

if one assumes $1 \ll N_{i} \ll G_{i}$ and negligibly correlated distributions. The total phase space volume is

$$
\Delta \Gamma=\prod_{i} \Delta \Gamma_{i}
$$

and the definition of entropy is $S=\ln \Delta \Gamma$. Using the approximation $\ln N ! \approx N \ln (N / e)$, we can express the entropy as

$$
\begin{aligned}
S & =\sum_{i} G_{i} \frac{N_{i}}{G_{i}} \ln \left[\frac{e G_{i}}{N_{i}}\right] \\
& \approx \int \frac{d^{3} p d^{3} x}{(2 \pi)^{3}} f(p, x) \ln \left[\frac{e}{f(p, x)}\right]
\end{aligned}
$$

where

$$
n(x) \equiv \int \frac{d^{3} p}{(2 \pi)^{3}} f(p, x)
$$

gives the number density of particles. Hence, one can define the entropy current

$$
s^{\mu} \equiv \int \frac{d^{3} p}{(2 \pi)^{3}} f(p, x) \ln \left[\frac{e}{f(p, x)}\right] \frac{p^{\mu}}{p^{0}}
$$

where we define the entropy density as usual as

$$
s \equiv s^{0} .
$$

Now, using the metric

$$
d s^{2}=d t^{2}-a^{2}(t) d \vec{x}^{2}
$$

one has

$$
s_{; \mu}^{\mu}=\dot{s}+3 H s
$$

and hence the entropy density Boltzmann equation becomes

$$
\dot{s}+3 H s=-\sum_{f} \sum_{i} \int d \Gamma C_{i}^{f}\left[f_{f}\right] \ln \left[f_{f}\right]
$$

where $C_{i}^{f}$ corresponds to $i$ th collision operator for the particle species $f$ in the Boltzmann equation and $d \Gamma$ represents the appropriate momentum phase space integral. Neglecting $C P$ violations which are not important for the total entropy generation, we can write the collision terms in terms of S-matrix amplitudes as

$$
\begin{aligned}
-\sum_{f} \sum_{i} \int d \Gamma C_{i}^{f}\left[f_{f}\right] \ln \left[f_{f}\right]= & \sum_{i} \int d \Gamma(2 \pi)^{4} \delta^{(4)}(\text { momenta }) \times\left(f_{a_{i}} f_{b_{i}}-f_{c_{i}} f_{d_{i}}\right)\left|M\left(c_{i}, d_{i} ; a_{i}, b_{i}\right)\right|^{2} \ln \left[\frac{f_{a_{i}} f_{b_{i}}}{f_{c_{i}} f_{d_{i}}}\right] \\
& +\sum_{i} \int d \Gamma(2 \pi)^{4} \delta^{(4)}(\text { momenta }) \times\left(f_{e_{i}}-f_{g_{i}} f_{h_{i}}\right)\left|M\left(g_{i}, h_{i} ; e_{i}\right)\right|^{2} \ln \left[\frac{f_{e_{i}}}{f_{g_{i}} f_{h_{i}}}\right] \\
& \times \sum_{i} \int d \Gamma(2 \pi)^{4} \delta^{(4)}(\text { momenta }) \times(\text { initial states }- \text { final states })|M|^{2} \\
& \times \ln \left[\frac{\text { initial states }}{\text { final states }}\right]+\cdots+\Delta C
\end{aligned}
$$


where the phase space integral

$$
d \Gamma \equiv \prod \frac{g}{(2 \pi)^{3}} \frac{d^{3} p}{2 E}
$$

is over all particles and momenta, and $\Delta C$ corresponds to the entropy change produced by the decay of a classical field configuration. If a zero-temperature classical field configuration (such as an inflaton VEV) decays to particles $X_{1}$ and $X_{2}$ and the Bogoliubov coefficient for the particle production is $\left|\beta_{k}\right|^{2}$ (where the physical momentum is $k / a$ ), then we have the effective collision operator

$$
\begin{aligned}
\Delta C= & \int d \Gamma C_{i}^{X_{1}}\left[f_{X_{1}}\right] \ln \left[f_{X_{1}}\right] \\
& +\int d \Gamma C_{i}^{X_{2}}\left[f_{X_{2}}\right] \ln \left[f_{X_{2}}\right] \\
= & -\int \frac{d^{3} p}{(2 \pi)^{3}} \ln \left[f_{X_{1}} f_{X_{2}}\right] \frac{d}{d t}\left|\beta_{p a}\right|^{2}
\end{aligned}
$$

contributing to the entropy density Boltzmann equation.

With our expression for the entropy, one can prove the following little theorem: If baryons are generated through particle $f$ which is out of equilibrium undergoing arbitrary reactions, then

$$
n_{B} \leqslant \frac{2 \eta}{a^{3}} \int d t \Gamma_{\mathrm{BV}}(t) a^{3} n_{f},
$$

and

$$
\frac{n_{B}}{s}<2 \eta\left[\frac{\Gamma_{\mathrm{BV}}(t)}{\Gamma_{\mathrm{t}}(t)}\right]_{\max }
$$

where

$$
\eta \equiv \alpha_{W} \sum_{i j} I \operatorname{Im}\left\{\lambda^{a j b i} \lambda^{* a t b s} V_{i s} V_{t j}^{*}\right\} \sim 0.02 \frac{\bar{T}^{2}}{v^{2}}
$$

$\bar{T}$ is the effective energy scale which characterizes the kinetic energy of the on shell particles in the loop [40], $I$ is the loop integral suppression factor, $v$ is the Higgs VEV of about 180 $\mathrm{GeV}$, and $\Gamma_{\mathrm{BV}}$ is the tree-level baryon number violating reaction rate. The maximum in Eq. (C13) is taken during the period when most of the baryogenesis is taking place, and $\Gamma_{\mathrm{t}}$ corresponds to the reaction rate of $f$ which does not violate baryon number.

The argument validating this claim goes as follows. Starting from Eq. (27), one can use the momentum conserving $\delta$-function and the form of the equilibrium distribution to obtain Eq. (C12) if one assumes that the baryon-numberviolating operator coupling is of order 1 (maximal). Now, consider the $f$ evolution. Defining $N_{f} \equiv n_{f} a^{3}$, we can write the $B$-conserving part of the Boltzmann equation as

$$
\dot{N}_{f}=-\sum_{i} \Gamma^{i}\left(N_{f}-N_{f}^{e q} U_{f}^{i}\right)+\Delta C a^{3}+\cdots
$$

where $\Gamma^{i}$ are baryon number-conserving reaction rates, $N_{f}^{e q}$ are equilibrium number densities per comoving volume, $U_{f}^{i}$ are the ratios of final state number densities to initial state equilibrium number densities that usually appear in the Boltzmann equations, and $\Delta \mathrm{Ca}^{3}$ is the external source for $f$. Since we are assuming maximal out of equilibrium, the equilibrium terms involving reverse reactions can be neglected. Hence, we can write

$$
\dot{N}_{f} \approx-\Gamma_{\mathrm{t}}(t) N_{f}+\Delta C a^{3}
$$

which governs $N_{f}$ neglecting the baryon number violating terms. Hence, we can write

$$
n_{B} \leqslant \frac{2 \eta}{a^{3}} \int_{t_{i}}^{t} d t^{\prime} \frac{\Gamma_{\mathrm{BV}}\left(t^{\prime}\right)}{\Gamma_{\mathrm{t}}\left(t^{\prime}\right)}\left(\Delta C\left(t^{\prime}\right) a^{3}\left(t^{\prime}\right)-\dot{N}_{f}\left(t^{\prime}\right)\right) .
$$

Furthermore, we can write

$$
\begin{aligned}
n_{B} \leqslant & \frac{2 \eta}{a^{3}}\left[\frac{\Gamma_{\mathrm{BV}}}{\Gamma_{\mathrm{t}}}\right]_{\max }\left(\int_{t_{i}}^{t} d t^{\prime} \Delta C\left(t^{\prime}\right) a^{3}\left(t^{\prime}\right)\right. \\
& \left.-N_{f}(t)+N_{f}\left(t_{i}\right)\right) .
\end{aligned}
$$

From Eq. (C8), we see that

$$
s a^{3} \geqslant N_{f}\left(t_{i}\right)+\int_{t_{i}}^{t} d t^{\prime} \Delta C\left(t^{\prime}\right) a^{3}\left(t^{\prime}\right) .
$$

Hence, we find Eq. (C13), as promised.

\section{APPENDIX D: SCALING OF TEMPERATURE}

Here we discuss the physical range of $\nu$ in $T \propto a^{-\nu}$. The generalized first law of thermodynamics tells us

$$
d E=-P d V+T d S+J_{\rho} d t
$$

where $J_{\rho}$ is the external source or sink of energy for the photons and for other relativistic fluids in thermal equilibrium with the photons. Here we have separated the 3 dimensional pressure $P$ from the component associated with the extra dimension, whose contribution to $d E$ we write as $J_{\rho} d t$. If we assume a constant "effective" average equation of state $P / \rho \approx \omega$, set $d S=0$, and assume that $d V=a^{3}$, then we find, trivially,

$$
\frac{\rho}{\rho_{i}}=\exp \left(\int \frac{J_{\rho}}{a^{3} \rho} d t\right)\left(\frac{a}{a_{i}}\right)^{-3(\omega+1)} .
$$

For photons and other relativistic particles in thermal equilibrium confined to 3 spatial dimensions, the equation of state is $\omega \approx 1 / 3$ and $\rho \propto T^{4}$. This implies 


$$
T=T_{i} \exp \left(\frac{1}{4} \int \frac{J_{\rho}}{a^{3} \rho} d t\right)\left(\frac{a}{a_{i}}\right)^{-1}
$$

which implies that $\nu=1$ unless the source term $\int d t\left(J_{\rho} / a^{3} \rho\right)$ is significant. If $J_{\rho}>0$, then $\nu<1$, otherwise $\nu>1$.

An example of a situation in which $\nu<1$ is when the bulk fields are in thermal equilibrium with the brane fields through

$$
J_{\rho} d t \approx-P_{4+d} a^{3} d z^{d}
$$

where $P_{5}$ is the bulk pressure and $z$ is the scale factor for the extra dimension.
[1] J.D. Lykken, Phys. Rev. D 54, 3693 (1996).

[2] N. Arkani-Hamed, S. Dimopoulos, and G.R. Dvali, Phys. Lett. B 429, 263 (1998); Phys. Rev. D 59, 086004 (1999); I. Antoniadis, N. Arkani-Hamed, S. Dimopoulos, and G.R. Dvali, Phys. Lett. B 436, 257 (1998).

[3] T. Banks and L.J. Dixon, Nucl. Phys. B307, 93 (1988); G. Gilbert, ibid. B328, 159 (1989).

[4] Particle Data Group, D.E. Groom et al., Eur. Phys. J. C 15, 1 (2000).

[5] K. Benakli and S. Davidson, Phys. Rev. D 60, 025004 (1999).

[6] N. Arkani-Hamed and M. Schmaltz, Phys. Rev. D 61, 033005 (2000).

[7] G. Aldazabal, S. Franco, L.E. Ibáñez, R. Rabadan, and A.M. Uranga, J. High Energy Phys. 02, 047 (2001); L.E. Ibáñez, F. Marchesano, and R. Rabadan, ibid. 11, 002 (2001).

[8] L.J. Hall and D.R. Smith, Phys. Rev. D 60, 085008 (1999); M. Fairbairn, Phys. Lett. B 508, 335 (2001); S. Hannestad, Phys. Rev. D 64, 023515 (2001).

[9] M. Fairbairn and L.M. Griffiths, J. High Energy Phys. 02, 024 (2002).

[10] S. Cullen and M. Perelstein, Phys. Rev. Lett. 83, 268 (1999); V. Barger, T. Han, C. Kao, and R.J. Zhang, Phys. Lett. B 461, 34 (1999); S. Hannestad and G. Raffelt, Phys. Rev. Lett. 87, 051301 (2001); 88, 071301 (2002).

[11] G.F. Giudice, R. Rattazzi, and J.D. Wells, Nucl. Phys. B544, 3 (1999); E.A. Mirabelli, M. Perelstein, and M.E. Peskin, Phys. Rev. Lett. 82, 2236 (1999); T. Han, J.D. Lykken, and R.J. Zhang, Phys. Rev. D 59, 105006 (1999); J.L. Hewett, Phys. Rev. Lett. 82, 4765 (1999); I. Antoniadis, Phys. Lett. B 246, 377 (1990); I. Antoniadis, K. Benakli, and M. Quiros, ibid. 331, 313 (1994); 460, 176 (1999); P. Nath and M. Yamaguchi, Phys. Rev. D 60, 116004 (1999); M. Masip and A. Pomarol, ibid. 60, 096005 (1999); P. Nath, Y. Yamada, and M. Yamaguchi, Phys. Lett. B 466, 100 (1999); T.G. Rizzo and J.D. Wells, Phys. Rev. D 61, 016007 (2000); C.D. Carone, ibid. 61, 015008 (2000).

[12] L.M. Krauss and F. Wilczek, Phys. Rev. Lett. 62, 1221 (1989).

[13] R. Blumenhagen, B. Körs, D. Lüst, and T. Ott, Nucl. Phys. B616, 3 (2001); R. Blumenhagen, B. Körs, and D. Lüst, J. High Energy Phys. 02, 030 (2001); R. Blumenhagen, L. Görlich, B. Körs, and D. Lüst, ibid. 10, 006 (2000).

[14] S. Davidson, M. Losada, and A. Riotto, Phys. Rev. Lett. 84, 4284 (2000); G.R. Dvali and G. Gabadadze, Phys. Lett. B 460, 47 (1999).

[15] It is unclear whether electroweak baryogenesis with low $T_{\text {rh }}$ ([14], first reference) actually satisfies the bounds, since a hot plasma at temperature $T \gg T_{\mathrm{rh}}$ is needed.
[16] K.R. Dienes, E. Dudas, and T. Gherghetta, Nucl. Phys. B537, 47 (1999).

[17] Assuming that no light fermions with $B$-violating interactions propagate over the bulk.

[18] F.C. Adams, G.L. Kane, M. Mbonye, and M.J. Perry, Int. J. Mod. Phys. A 16, 2399 (2001).

[19] A. Masiero, M. Peloso, L. Sorbo, and R. Tabbash, Phys. Rev. D 62, 063515 (2000).

[20] R. Allahverdi, K. Enqvist, A. Mazumdar, and A. PerezLorenzana, Nucl. Phys. B618, 277 (2001); A. Mazumdar and A. Perez-Lorenzana, Phys. Rev. D 65, 107301 (2002).

[21] A.D. Sakharov, Pis'ma Zh. Éksp. Teor. Fiz. 5, 32 (1967) [JETP Lett. 5, 24 (1967)].

[22] K. Akama, in Pregeometry, Lecture Notes in Physics Vol. 176, edited by K. Kikkawa, N. Nakanishi, and H. Nariai (SpringerVerlag, Berlin, 1982), p. 267; V.A. Rubakov and M.E. Shaposhnikov, Phys. Lett. 125B, 136 (1983).

[23] H. Georgi, A.K. Grant, and G. Hailu, Phys. Rev. D 63, 064027 (2001).

[24] We also assume that the brane tension is negligible such that Randall-Sundrum type of warping is negligible.

[25] It is typically somewhere between 70 and $180 \mathrm{GeV}$ for the standard thermal scenario.

[26] A.D. Dolgov, Pis'ma Zh. Eksp. Teor. Fiz. 29, 254 (1979).

[27] E.W. Kolb and S. Wolfram, Nucl. Phys. B172, 224 (1980); B195, 542(E) (1980).

[28] Note that no color factors were included.

[29] Again, the reader is reminded that by temperature, here we mean a scale for particle density rather than implying that the particles are truly in equilibrium.

[30] By considering the mixing of $\nu_{\tau}$, we are assuming that the neutrinos have mass. In that case, there may generically be scalars that can produce dimension-5 operators. However, if these scalars are sufficiently heavy, then their contribution will be negligible. We do not analyze such cases for which beyond the standard model particles actively participate in the short distance physics.

[31] R.J. Crewther, J. Finjord, and P. Minkowski, Nucl. Phys. B207, 269 (1982).

[32] At the one loop level, there is a nonlocal contribution to $\nu \bar{\nu}$ $\rightarrow \gamma \gamma$ that is nonzero even in the zero neutrino mass limit [31].

[33] It is trivial to show that particle $X$ cannot have approximately equilibrium distribution if the particles with which $X$ interacts most strongly are not in equilibrium.

[34] V.A. Kuzmin, V.A. Rubakov, and M.E. Shaposhnikov, Phys. Lett. B 155, 36 (1985).

[35] V.A. Rubakov and M.E. Shaposhnikov, Usp. Fiz. Nauk 166, 493 (1996) [Phys. Usp. 39, 461 (1996)]. 
[36] The mixed $\mathrm{U}(1)_{B} \times \mathrm{U}(1)_{Y}^{2}$ anomaly is also nonvanishing, but there are no nontrivial U(1) gauge configurations analogous to the instanton or sphaleron.

[37] C.T. Hill and P. Ramond, Nucl. Phys. B596, 243 (2001).

[38] Hill and Ramond have reinterpreted the four-dimensional instanton rate as the amplitude for a massive soliton in fivedimensional gauge theory to propagate over the entire fifth dimension [37].

[39] Unless a lepton asymmetry associated with the conserved $B-L$ number is generated at high scales.

[40] More specifically, $\bar{T}^{2}$ is approximately $T_{\text {init }}^{2}+m_{\text {init }}^{2}-\left(m_{u i}\right.$ $\left.+m_{d j}\right)^{2}$ where $T_{\text {init }}$ and $m_{\text {init }}$ are temperature and mass scales of the initial reactants and $m_{u i}$ and $m_{d j}$ are the masses of the particles in the loop. 